\title{
Measuring localized nonlinear components in a circular accelerator with a nonlinear tune response matrix
}

\author{
G. Franchetti, A. Parfenova, and I. Hofmann \\ GSI Darmstadt, D-64291 Darmstadt, Germany \\ (Received 2 May 2008; published 16 September 2008)
}

\begin{abstract}
In this paper we present a method for measuring the nonlinear errors in a circular accelerator by taking advantage of the feed-down effect of high order multipoles when the closed orbit is globally deformed. We devise a nonlinear tune response matrix in which the response to a closed orbit deformation is obtained in terms of change of machine tune and correlated with the strength of the local multipoles. A numerical example and a proof of principle experiment to validate the theoretical methods are presented and discussed.
\end{abstract}

DOI: 10.1103/PhysRevSTAB.11.094001

PACS numbers: 41.85.Lc, 29.27.Bd

\section{INTRODUCTION}

The requirements of beam control for operation in synchrotrons necessitate that an accelerator working point is sufficiently far from any machine resonance. Errors deriving from higher order field components of magnets are the major source for the excitation of nonlinear resonances. When hardware constraints do not allow to change arbitrarily the machine working point, it may be necessary to compensate some of the resonances present in the machine in order to maintain a high accelerator performance. The presence of space charge makes the role of nonlinear resonances more central in case of the storage of a high intensity bunched beam. In fact, the overlapping of the space charge tune spread with a machine nonlinear resonance leads to long term beam loss [1]. In this case resonance compensation has to be carefully discussed as the presence of space charge may influence the effectiveness of the compensation system.

A general treatment of resonances in accelerators with perturbation theory [2] or via normal form [3] shows that the excitation of a resonance can be correlated to resonance driving terms, which depend on the nonlinear error distribution around the ring. The consequences of the nonlinear errors in terms of a change of the beam distribution and of beam loss are not easy to predict and benchmark with respect to measurements [4-6]. An attempt to cover the bridge between the complexity of the theory and the necessity of devising a method to measure nonlinear errors in an existing accelerator is described in [7] via turn by turn data acquisition. Later this approach was applied to the normal form [8], and further progress in measuring errors in circular accelerator is reported in Ref. [9]. All these methods rely on the high precision in the measurement of tunes made possible with the advent of the methods developed in Ref. [10]. Recently, another method applicable to systematic errors was obtained via the measurement of the nonlinear chromaticity: in this method the energy of a well controlled bunch is slightly increased and $Q_{x}, Q_{y}$ is measured versus $\delta p / p$. For these measured tunes the effect of the nonlinearities is folded in globally, and these data can be used to retrieve a theoretical model of the accelerator. In fact, in a computer model the free parameters for creating the nonlinear chromaticity are the strength of the nonlinear systematic errors, which are assumed localized in dipoles and quadrupoles, therefore the best fitting of the numerical nonlinear chromaticity with the measured chromaticity allows the setting of the systematic nonlinear errors in magnets [11,12]. The analytic calculation of the detuning for an off-energy closed orbit can be computed at any order with a differential algebra-based method described in Ref. [13]. This technique incorporates automatically the correct closed orbit and the effect of nonlinear errors on the chromaticity. As the off-energy closed orbit provides only one free parameter to reconstruct the effect of localized nonlinear errors, an alternative approach presented in Ref. [14] uses local orbit bumps. The beam is moved locally via orbit bumps and the detuning induced by feed-down is the observable, which contains the effect of the nonlinearities. In Ref. [14] this method is applied to the RHIC interaction region. These authors, however, found a difficulty in retrieving skew components for sextupolar and octupolar nonlinear errors. In a later work, in Ref. [15], this difficulty is avoided by equally deforming the closed orbit in both horizontal and vertical planes. This approach works well when the local bump acts only on one error. The simultaneous presence of sextupoles and octupoles creates a linear coupling, which is difficult to remove as it would require a very special correlation between horizontal and vertical closed orbit deformations. Therefore the simultaneous presence of several nonlinear errors in regions where the closed orbit is deformed requires the assessment of the detuning induced by the feed-down linear coupling.

In this paper we extend the strategy initiated in Ref. [14] and extended in Ref. [15] by releasing the local bump restriction: the closed orbit is globally deformed by all 
the accelerator steerers obtaining a detuning dependence which is a quadratic form of all the steerer strength and also a quadratic form of the strength of the nonlinear errors. This approach has an analogy with the linear orbit response matrix method. In our approach we explicitly calculate the perturbative contribution of the skew linear components induced by the deformation of the closed orbit. The analytic results allow us to reconstruct sextupolar and octupolar normal and skew errors in a circular accelerator. The plan of the paper is the following: In Sec. II we present the theoretical basis of the method; in Sec. III we show an application of this method to the SIS18 and discuss via numerical simulations the robustness and the limits of the method proposed; in Sec. IV we present the measurement results performed in the SIS18 synchrotron in which we made a proof of principle experiment for demonstrating that this method can be used in a real synchrotron. Section V is devoted to the conclusion and the outlook and in the Appendix we discuss the more technical mathematical aspects of the derivation of this method.

\section{THEORETICAL BASIS OF THE NONLINEAR TUNE RESPONSE MATRIX METHOD}

This approach has an analogy with the method developed in the orbit response matrix (ORM) [16], where the local orbit response, i.e., the orbit deformation as function of the steerer value, is measured and information on the linear optics can be retrieved. In particular, a fitting of the ORM performed with computer models allows one to obtain the best modeling of a real accelerator. The method discussed here extends the ORM method to the changes in tunes created by nonlinear components feed-down. We refer to this method as nonlinear tune response matrix (NTRM) in analogy with the ORM.

\section{A. Linear and nonlinear accelerator model}

We consider a linear model of a circular accelerator composed by a sequence of linear thick elements as drifts, quadrupoles, and dipoles and assume tunes sufficiently far off a resonance. The strengths of the linear focusing forces is defined by $k_{x}(s), k_{y}(s)$, where $s$ is the longitudinal coordinate. We assume that the accelerator is equipped with $N_{t}$ thin steerers each of which can act independently in the horizontal and vertical plane. The longitudinal location of the $t$ th steerer is $s_{t}$, and its steering angle is $\theta_{x t}$ in the horizontal plane, $\theta_{y t}$ in the vertical plane. We also include in the ring $N_{l}$ thin nonlinear elements. A nonlinear element can be a lattice sextupole or octupole as well as a magnet nonlinear error. In general, the $l$ th nonlinear error located at $s_{l}$ is composed of several multipoles of integrated strength $K_{n l}, J_{n l}, n \geq 1$. Here the index $n$ is used for indicating the order of the nonlinear component, and $l$ is the location as shown in Fig. 1. In a real accelerator some of these errors may be negligible. With these definitions the singleparticle equation of motion reads

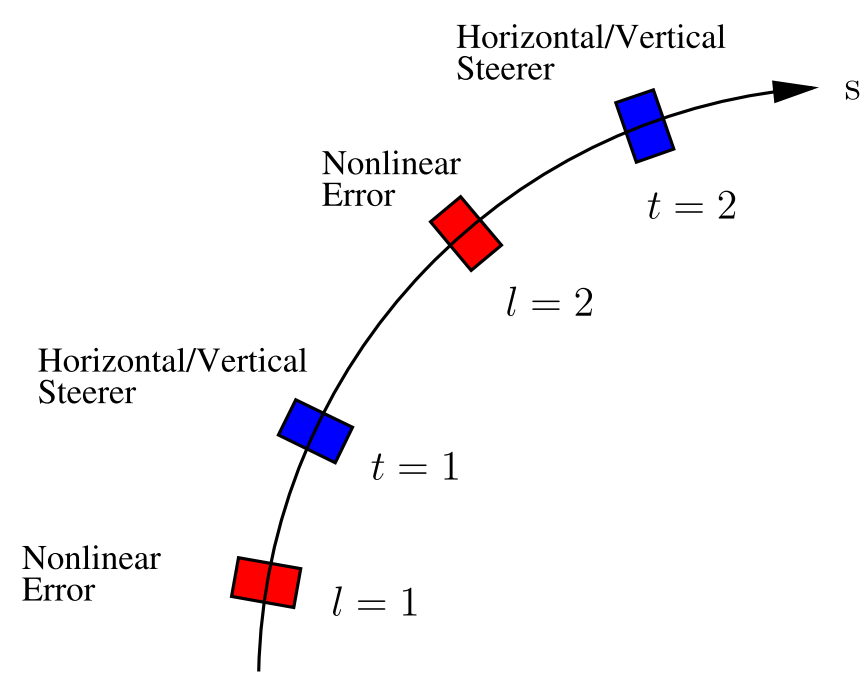

FIG. 1. (Color) Schematic of the nonlinear error and steerer locations.

$$
\begin{aligned}
& x^{\prime \prime}+k_{x} x=-\operatorname{Re}\left[\sum_{n \geq 1} \frac{k_{n}+i j_{n}}{n !}(x+i y)^{n}\right]+\theta_{x} \\
& y^{\prime \prime}+k_{y} y=\operatorname{Im}\left[\sum_{n \geq 1} \frac{k_{n}+i j_{n}}{n !}(x+i y)^{n}\right]+\theta_{y}
\end{aligned}
$$

with ()$^{\prime}=d() / d s$, and

$$
\begin{array}{rlrl}
\theta_{x} & =\sum_{t=1}^{N_{t}} \theta_{x t} \delta\left(s-s_{t}\right), & \theta_{y} & =\sum_{t=1}^{N_{t}} \theta_{y t} \delta\left(s-s_{t}\right), \\
k_{n}=\sum_{l=1}^{N_{l}} K_{n l} \delta\left(s-s_{l}\right), & j_{n}=\sum_{l=1}^{N_{l}} J_{n l} \delta\left(s-s_{l}\right) .
\end{array}
$$

All the quantities $k_{x}, k_{y}, k_{n}, j_{n}, \theta_{x}, \theta_{y}$ are $s$ dependent, but for simplicity of notation we omit this dependence. Note that Eqs. (1) are written with respect to the reference closed orbit, which is identified here by $x=0, y=0$ when $\theta_{x}=$ $\theta_{y}=0$.

\section{B. The distorted closed orbit}

We now deform the closed orbit by setting the $N_{t}$ steering angles $\theta_{x t}, \theta_{y t}$, with $t=1, \ldots, N_{t}$ to a value different from zero. The new closed orbit $\left(x_{o}, x_{o}^{\prime}, y_{o}, y_{o}^{\prime}\right)$ is the solution of the equations

$$
\begin{aligned}
& x_{o}^{\prime \prime}+k_{x} x_{o}=-\operatorname{Re}\left[\sum_{n \geq 1} \frac{k_{n}+i j_{n}}{n !}\left(x_{o}+i y_{o}\right)^{n}\right]+\theta_{x}, \\
& y_{o}^{\prime \prime}+k_{y} y_{o}=\operatorname{Im}\left[\sum_{n \geq 1} \frac{k_{n}+i j_{n}}{n !}\left(x_{o}+i y_{o}\right)^{n}\right]+\theta_{y},
\end{aligned}
$$

with the boundary condition $x_{o}(s)=x_{o}(s+C), x_{o}^{\prime}(s)=$ $x_{o}^{\prime}(s+C), y_{o}(s)=y_{o}(s+C), y_{o}^{\prime}(s)=y_{o}^{\prime}(s+C)$ for $0 \leq$ $s \leq C$, where $C$ stands for the length of the ring. If the closed orbit deformation is not too large and the tunes are not close to any relevant resonance, then the nonlinear 
components in Eqs. (3) do not play an important role in determining the closed orbit, which can be described by the equations

$$
x_{o}^{\prime \prime}+k_{x} x_{o}=\theta_{x}, \quad y_{o}^{\prime \prime}+k_{y} y_{o}=\theta_{y} .
$$

As Eqs. (4) are linear, the value of $x_{o}, y_{o}$ at a specific longitudinal location is a linear combination of the steering angles. At the location of the $l$ th nonlinear element $s_{l}$, we find that $x_{o l} \equiv x_{o}\left(s_{l}\right)$ and $y_{o l} \equiv y_{o}\left(s_{l}\right)$ are given by

$$
x_{o l}=\sum_{t=1}^{N_{t}} M_{l t}^{x} \theta_{x t}, \quad y_{o l}=\sum_{t=1}^{N_{t}} M_{l t}^{y} \theta_{y t} .
$$

The first index in these matrices refers to the location of the error, the second to the location of the steerer. The matrices $M^{x}, M^{y}$ form the orbit response matrix, $M=M^{x} \oplus M^{y}$, for the decoupled system. Note that for $N_{t}=N_{l}$, the invertibility of the matrices $M_{l t}^{x}$ and $M_{l t}^{y}$ depends on the lattice: in fact if $M_{l t}^{x}$ were not invertible, there would be two different configurations of $\theta_{x t}$, say $\theta_{x t}^{(1)}$ and $\theta_{x t}^{(2)}$, such that $x_{o l}=$ $\sum_{t=1, N_{t}} M_{l t}^{x} \theta_{x t}^{(1)}=\sum_{t=1, N_{t}} M_{l t}^{x} \theta_{x t}^{(2)}$. Therefore there would exist a steerer configuration $\theta_{x t}^{*}=\theta_{x t}^{(1)}-\theta_{x t}^{(2)}$ such that $\sum_{t=1, N_{t}} M_{l t}^{x} \theta_{x t}^{*}=0$ for every $l$. But such a configuration of the steerers, $\theta_{x t}^{*}$, is possible only if the location of the steerers $s_{t}$ and locations of the errors $s_{l}$ satisfy some very special condition. For instance, for a lattice with the sequence of steerers alternated with lattice nonlinear errors, such a condition reads

$$
1=(-1)^{N_{t}} \prod_{t=1}^{N_{t}} \frac{\sin \psi_{x t}}{\sin \hat{\psi}_{x t}}
$$

Here $\psi_{x t}$ is the phase advance between the $t$ th steerer located at $s=s_{t}$ and the $t$ th nonlinear error (the first nonlinear error after the $t$ th steerer); instead $\hat{\psi}_{x t}$ is the phase advance between the location of the $t$ th nonlinear error and the $(t+1)$ th steerer (i.e. the first steerer after the $t$ th nonlinear error). Note that the setting for the steerers $\theta_{x t}^{*}$ consistent with Eq. (6) depends on the angle in which the closed orbit passes through one multipolar error. As an example we assume the closed orbit passes through the first multipolar error with coordinates $\left(0, x_{1}^{\prime}\right)$ and there the beta function is $\beta_{x 1}$, then the configuration of steerers consistent with Eq. (6) is given by

$$
\theta_{x t}^{*}=x_{1}^{\prime} \sqrt{\frac{\beta_{x 1}}{\beta_{x t}}} \frac{\sin \left(\psi_{x t}+\hat{\psi}_{x t}\right)}{\sin \hat{\psi}_{x t}}(-1)^{t} \prod_{i=1}^{t-1} \frac{\sin \psi_{x i}}{\sin \hat{\psi}_{x i}},
$$

where $1 \leq t \leq N_{t}$, and $\beta_{x t}$ is the beta function at the location of the $t$ th steerer [for $t=1$ the $(t-1)$ fold product should be replaced by 1]. Note that if the number of errors is odd and $\psi_{x t}, \hat{\psi}_{x t}<\pi / 2$, then the matrix $M_{l t}^{x}$ is always invertible, because Eq. (6) can never be satisfied. A similar argument holds for $M^{y}$.

\section{Motion around the distorted closed orbit}

Consider now a test particle of coordinates $\left(\tilde{x}, \tilde{x}^{\prime}, \tilde{y}, \tilde{y}^{\prime}\right)$ with respect to the deformed closed orbit $\left(x_{o}, x_{o}^{\prime}, y_{o}, y_{o}^{\prime}\right)$. In the original reference frame this particle has coordinates $\left(x_{o}+\tilde{x}, x_{o}^{\prime}+\tilde{x}^{\prime}, y_{o}+\tilde{y}, y_{o}^{\prime}+\tilde{y}^{\prime}\right)$ and its motion is governed by

$$
\begin{aligned}
\left(x_{o}+\tilde{x}\right)^{\prime \prime}+k_{x}\left(x_{o}+\tilde{x}\right)= & -\operatorname{Re}\left\{\sum_{n \geq 1} \frac{k_{n}+i j_{n}}{n !}\right. \\
& \left.\times\left[\left(x_{o}+\tilde{x}\right)+i\left(y_{o}+\tilde{y}\right)\right]^{n}\right\}+\theta_{x}, \\
\left(y_{o}+\tilde{y}\right)^{\prime \prime}+k_{y}\left(y_{o}+\tilde{y}\right)= & \operatorname{Im}\left\{\sum_{n \geq 1} \frac{k_{n}+i j_{n}}{n !}\right. \\
& \left.\times\left[\left(x_{o}+\tilde{x}\right)+i\left(y_{o}+\tilde{y}\right)\right]^{n}\right\}+\theta_{y} .
\end{aligned}
$$

By expanding to the first order we find

$$
\begin{aligned}
{\left[\left(x_{o}+\tilde{x}\right)+i\left(y_{o}+\tilde{y}\right)\right]^{n}=} & \left(x_{o}+i y_{o}\right)^{n}+n\left(x_{o}+i y_{o}\right)^{n-1} \\
& \times(\tilde{x}+i \tilde{y})+O\left[(\tilde{x}+i \tilde{y})^{2}\right] .
\end{aligned}
$$

If the coordinates of the test particle $\left(\tilde{x}, \tilde{x}^{\prime}, \tilde{y}, \tilde{y}^{\prime}\right)$ are small, then all the terms of order higher than the first can be neglected as the tunes are far from any resonance. In this approximation the evolution of the particle coordinates $\tilde{x}, \tilde{y}$ with respect to the distorted closed orbit $\left(x_{o}, x_{o}^{\prime}, y_{o}, y_{o}^{\prime}\right)$ is given by the equations

$$
\begin{aligned}
& \tilde{x}^{\prime \prime}+k_{x} \tilde{x}=-\operatorname{Re}\left[\sum_{n \geq 1} \frac{k_{n}+i j_{n}}{(n-1) !}\left(x_{o}+i y_{o}\right)^{n-1}(\tilde{x}+i \tilde{y})\right], \\
& \tilde{y}^{\prime \prime}+k_{y} \tilde{y}=\operatorname{Im}\left[\sum_{n \geq 1} \frac{k_{n}+i j_{n}}{(n-1) !}\left(x_{o}+i y_{o}\right)^{n-1}(\tilde{x}+i \tilde{y})\right] .
\end{aligned}
$$

As all the relevant perturbative terms are linear, we can now redefine in Eqs. (10) the argument of the sum in terms of new gradient components as follows:

$$
\tilde{k}_{1}^{(n)}+i \tilde{j}_{1}^{(n)}=\frac{k_{n}+i j_{n}}{(n-1) !}\left(x_{o}+i y_{o}\right)^{n-1} .
$$

Note that all the components $\tilde{k}_{1}^{(n)}, \tilde{j}_{1}^{(n)}$ are localized components deriving from the localized components $k_{n}, j_{n}$ described by Eqs. (2). The first three orders of expansion of these components are listed in Table I.

TABLE I. Leading three orders of the feed-down due to the deformed closed orbit.

\begin{tabular}{lcc}
\hline \hline$n$ & $\tilde{k}_{1}^{(n)}$ & $\tilde{j}_{1}^{(n)}$ \\
\hline 1 & $k_{1}$ & $j_{1}$ \\
2 & $k_{2} x_{o}-j_{2} y_{o}$ & $k_{2} y_{o}+j_{2} x_{o}$ \\
3 & $\frac{1}{2} k_{3}\left(x_{o}^{2}-y_{o}^{2}\right)-j_{3} x_{o} y_{o}$ & $k_{3} x_{o} y_{o}+\frac{1}{2} j_{3}\left(x_{o}^{2}-y_{o}^{2}\right)$ \\
\hline \hline
\end{tabular}


The final form of the equations of motion for a particle with small amplitude in an accelerator with a deformed closed orbit reads

$$
\tilde{x}^{\prime \prime}+\left(k_{x}+\tilde{k}\right) \tilde{x}=\tilde{j} \tilde{y}, \quad \tilde{y}^{\prime \prime}+\left(k_{y}-\tilde{k}\right) \tilde{y}=\tilde{j} \tilde{x},
$$

where

$$
\tilde{k}=\sum_{n \geq 1} \tilde{k}_{1}^{(n)}, \quad \tilde{j}=\sum_{n \geq 1} \tilde{j}_{1}^{(n)} .
$$

From Eqs. (12) we find that the nonlinear components around the ring produce an extra linear focusing component of strength $\tilde{k}$ and a linear coupling term of strength $\tilde{j}$. Summing up the first order components of Table I we find $\tilde{k}=k_{1}+k_{2} x_{o}-j_{2} y_{o}+k_{3} \frac{1}{2}\left(x_{o}^{2}-y_{o}^{2}\right)-j_{3} x_{o} y_{o}$,

$\tilde{j}=j_{1}+k_{2} y_{o}+j_{2} x_{o}+k_{3} x_{o} y_{o}+j_{3} \frac{1}{2}\left(x_{o}^{2}-y_{o}^{2}\right)$.

For example, if the closed orbit is deformed only in the vertical plane, we find

$$
\tilde{k}=k_{1}-j_{2} y_{o}-\frac{k_{3} y_{o}^{2}}{2}, \quad \tilde{j}=j_{1}+k_{2} y_{o}-\frac{j_{3} y_{o}^{2}}{2} .
$$

Limiting to normal sextupolar and normal octupolar components, we find

$$
\tilde{k}=-\frac{k_{3} y_{o}^{2}}{2}, \quad \tilde{j}=k_{2} y_{o} .
$$

Normal sextupolar components create linear coupling if the closed orbit is vertically deformed.

\section{Consequences of the feed-down induced linear gradient components}

When only small gradient errors $\tilde{k}$ are present, and the machine tunes are far from any integer or half integer resonance, the tunes with respect to the distorted closed orbit $\left(x_{o}, x_{o}^{\prime}, y_{o}, y_{o}^{\prime}\right)$, can be evaluated via the formulas [17]

$$
\begin{aligned}
& \Delta Q_{x}=\frac{1}{4 \pi} \int_{0}^{C} \beta_{x}(s) \tilde{k}(s) d s, \\
& \Delta Q_{y}=-\frac{1}{4 \pi} \int_{0}^{C} \beta_{y}(s) \tilde{k}(s) d s,
\end{aligned}
$$

where the tunes with respect to the closed orbit are defined by $Q_{x}=Q_{x 0}+\Delta Q_{x}$, and $Q_{y}=Q_{y 0}+\Delta Q_{y}$. Here $Q_{x 0}$, $Q_{y 0}$ are the tunes of the linear accelerator with the closed orbit corrected. These equations represent the first order contribution of the distribution of small gradient $\tilde{k}$ along the accelerator circumference. If the perturbative condition is not fulfilled, or if the sum gives a too small value of $\Delta Q_{x}$, then the higher order terms, quadratic in $\tilde{k}$, should be taken into account (see [17]). This is the case for the linear error described by Eqs. (14), where in addition the deformed closed orbit creates also localized skew linear errors due to feed-down of nonlinear components. Clearly, the presence of the skew gradient brings extra complications as the tunes $Q_{x}, Q_{y}$ in this case are found as coupled normal modes of the one-turn map $\mathbf{M}_{T}$ of the circular accelerator. Through a lengthy matrix algebra described in Appendix A, under the assumptions $J_{1 l} \sim 0$, and $\left|K_{1 l}\right| \ll$ $\left|K_{2 l}\right|,\left|J_{2 l}\right|,\left|K_{3 l}\right|,\left|J_{3 l}\right|$, we find that the detuning of a particle close to a deformed orbit created by $N_{t}$ steerers is given by

$$
\begin{aligned}
& \Delta Q_{x}={ }_{x} Q+\sum_{t=1}^{N_{t}}\left({ }_{x} Q_{t}^{x} \theta_{x t}+{ }_{x} Q_{t}^{y} \theta_{y t}\right) \\
&+\sum_{t, i=1}^{N_{t}}\left({ }_{x} Q_{t i}^{x x} \theta_{x t} \theta_{x i}+{ }_{x} Q_{t i}^{y y} \theta_{y t} \theta_{y i}+{ }_{x} Q_{t i}^{x y} \theta_{x t} \theta_{y i}\right), \\
& \Delta Q_{y}={ }_{y} Q+\sum_{t=1}^{N_{t}}\left({ }_{y} Q_{t}^{x} \theta_{x t}+{ }_{y} Q_{t}^{y} \theta_{y t}\right) \\
&+\sum_{t, i=1}^{N_{t}}\left({ }_{y} Q_{t i}^{x x} \theta_{x t} \theta_{x i}+{ }_{y} Q_{t i}^{y y} \theta_{y t} \theta_{y i}+{ }_{y} Q_{t i}^{x y} \theta_{x t} \theta_{y i}\right),
\end{aligned}
$$

where

$$
\begin{aligned}
{ }_{x} Q= & \sum_{l=1}^{N_{l}} K_{1 l} \tilde{\mathcal{K}}_{l}^{x}+\sum_{l, q=1}^{N_{l}} K_{1 l} K_{1 q} \tilde{\mathcal{K}}_{l q}^{x}, \\
{ }_{x} Q_{t}^{x}= & \sum_{l=1}^{N_{l}} K_{2 l} \tilde{\mathcal{K}}_{l}^{x} M_{l t}^{x}, \\
{ }_{x} Q_{t}^{y}= & -\sum_{l=1}^{N_{l}} J_{2 l} \tilde{\mathcal{K}}_{l}^{x} M_{l t}^{y}, \\
{ }_{x} Q_{t i}^{x x}= & \frac{1}{2} \sum_{l=1}^{N_{l}} K_{3 l} \tilde{\mathcal{K}}_{l}^{x} M_{l l}^{x} M_{l i}^{x} \\
& +\sum_{l, q=1}^{N_{l}}\left(J_{2 l} J_{2 q} \tilde{\mathcal{J}}_{l q}^{x}+K_{2 l} K_{2 q} \tilde{\mathcal{K}}_{l q}^{x}\right) M_{l t}^{x} M_{q i}^{x}, \\
{ }_{x} Q_{t i}^{y y}= & -\frac{1}{2} \sum_{l=1}^{N_{l}} K_{3 l} \tilde{\mathcal{K}}_{l}^{x} M_{l t}^{y} M_{l i}^{y} \\
& +\sum_{l, q=1}^{N_{l}}\left(K_{2 l} K_{2 q} \tilde{\mathcal{J}}_{l q}^{x}+J_{2 l} J_{2 q} \tilde{\mathcal{K}}_{l q}^{x}\right) M_{l t}^{y} M_{q i}^{y}, \\
{ }_{x} Q_{t i}^{x y}= & -\sum_{l=1}^{N_{l}} J_{3 l} \tilde{\mathcal{K}}_{l}^{x} M_{l t}^{x} M_{l i}^{y}+\sum_{l, q=1}^{N_{l}}\left[J_{2 l} K_{2 q}\left(\tilde{\mathcal{J}}_{l q}^{x}+\tilde{\mathcal{J}}_{q l}^{x}\right)\right. \\
& \left.-J_{2 q} K_{2 l}\left(\tilde{\mathcal{K}}_{l q}^{x}+\tilde{\mathcal{K}}_{q l}^{x}\right)\right] M_{l t}^{x} M_{q i}^{y} .
\end{aligned}
$$

The equivalent terms ${ }_{y} Q,{ }_{y} Q_{t}^{x},{ }_{y} Q_{t}^{y},{ }_{y} Q_{t i}^{x x},{ }_{y} Q_{t i}^{y y},{ }_{y} Q_{t i}^{x y}$ are obtained replacing $x$ with $y$ at the apex of $\tilde{\mathcal{K}}_{l}^{x}, \tilde{\mathcal{K}}_{l q}^{x}, \tilde{\mathcal{J}}_{l q}^{x}$. The explicit form of the terms $\tilde{\mathcal{K}}_{l}^{x}, \tilde{\mathcal{K}}_{l q}^{x}, \tilde{\mathcal{J}}_{l q}^{x}$, which depend only on the locations of the errors, locations of the steerers and the tunes is given by Eqs. (A23) in Appendix A. Therefore, when the linear machine is known, these terms are known as well. Note that the linear components $K_{1 l}$ contribute to the tune $Q_{x}, Q_{y}$ independently from how the closed orbit is deformed: in the derivation used in Sec. II B, Eqs. (5) are obtained when $J_{1 l}=0$ in order to avoid the presence of coupling matrices. The 
derivation of the tunes is still possible if $J_{1 l} \neq 0$, but to a price of a much higher level of complexity in the analytic solutions. We assume therefore that $J_{1 l}$ is small, or previously corrected (see in Ref. [18] for a discussion of the correction of the natural linear coupling).

Therefore the effective tune due to linear elements will be $Q_{x 0 \text {,eff }}=Q_{x 0}+{ }_{x} Q$ and $Q_{y 0 \text {,eff }}=Q_{y 0}+{ }_{y} Q$. Note also that the detuning in the horizontal and vertical planes depends linearly on $K_{2 l}, J_{2 l}$, and that the octupolar terms $K_{3 l}, J_{3 l}$ produce a detuning quadratic in $\theta_{x l}, \theta_{y j}$, with an additional second order contribution of the normal and skew second order components.

\section{E. Measurement of the nonlinear components via nonlinear tune response matrix}

From Eq. (18) we see that in general the detuning induced by nonlinear components in a deformed closed orbit is a quadratic form in $\theta_{x t}, \theta_{y t}$. We can therefore take advantage of Eq. (18) and devise a method to reconstruct the nonlinear errors in a ring. In fact, we can determine via experimental measurements $\Delta Q_{x \text {,eff }}=Q_{x}-Q_{x 0 \text {,eff }}$ as function of $\theta_{x t}, \theta_{y t}$. If the number of measurements is large enough, we can obtain the quantities ${ }_{x} Q_{t}^{x},{ }_{x} Q_{t}^{y},{ }_{x} Q_{t i}^{x x},{ }_{x} Q_{t i}^{y y}$, ${ }_{x} Q_{t i}^{x y}\left(t, i=1, \ldots, N_{t}\right)$ by fitting, which are the components of the nonlinear tune response matrix (NTRM). By specifying the assumed location of the nonlinear errors, we compute then the matrices $M_{l t}^{x}, M_{l t}^{y}$, and $\beta_{x l}, \beta_{y l}$. For the particular case of $N_{l}=N_{t}$, i.e., for a number of errors equal to the number of steerers, these matrices are in general invertible and we can finally solve the system of equations (20) in the unknown variables $K_{n l}, J_{n l}$, with $n=$ 2,3 , and $l=1, \ldots, N_{l}$. Note that the number of equations for the octupolar errors, for example ${ }_{x} Q_{t i}^{x x}$, is larger than the number of unknown nonlinear strengths $K_{3 l}$. In order to find the values $K_{3 l}$, if the nonlinear tune measurements are sufficiently precise, it is enough to determine ${ }_{x} Q_{t t}^{x x},{ }_{x} Q_{t t}^{y y}$, ${ }_{x} Q_{t t}^{x y}$, with $t=1, \ldots, N_{t}$. By inspecting Eq. (18) we see that, if $\Delta Q_{x, \text { eff }}=\Delta Q_{x}\left(\theta_{x t}, \theta_{y t}\right)-{ }_{x} Q$ and we measure with equal steerer angle $\left|\theta_{x t}\right|=\left|\theta_{y t}\right|=\bar{\theta}_{t}$, then

$$
\begin{aligned}
{ }_{x} Q_{t}^{x}= & \frac{1}{2 \bar{\theta}_{t}}\left[\Delta Q_{x, \mathrm{eff}}\left(\bar{\theta}_{t}, 0\right)-\Delta Q_{x, \mathrm{eff}}\left(-\bar{\theta}_{t}, 0\right)\right], \\
{ }_{x} Q_{t}^{y}= & \frac{1}{2 \bar{\theta}_{t}}\left[\Delta Q_{x, \mathrm{eff}}\left(0, \bar{\theta}_{t}\right)-\Delta Q_{x, \mathrm{eff}}\left(0,-\bar{\theta}_{t}\right)\right], \\
{ }_{x} Q_{t t}^{x x}= & \frac{1}{2 \bar{\theta}_{t}^{2}}\left[\Delta Q_{x, \mathrm{eff}}\left(\bar{\theta}_{t}, 0\right)+\Delta Q_{x, \mathrm{eff}}\left(-\bar{\theta}_{t}, 0\right)\right], \\
{ }_{x} Q_{t t}^{y y}= & \frac{1}{2 \bar{\theta}_{t}^{2}}\left[\Delta Q_{x, \mathrm{eff}}\left(0, \bar{\theta}_{t}\right)+\Delta Q_{x, \mathrm{eff}}\left(0,-\bar{\theta}_{t}\right)\right], \\
{ }_{x} Q_{t t}^{x y}= & \frac{1}{2 \bar{\theta}_{t}^{2}}\left[\Delta Q_{x, \mathrm{eff}}\left(\bar{\theta}_{t}, \bar{\theta}_{t}\right)+\Delta Q_{x, \mathrm{eff}}\left(-\bar{\theta}_{t},-\bar{\theta}_{t}\right)\right] \\
& -{ }_{x} Q_{t t}^{x x}-{ }_{x} Q_{t t}^{y y} .
\end{aligned}
$$

This then reduces the number of measurements needed for retrieving the $N_{l}$ nonlinear errors.

\section{APPLICATIONS AND LIMITS}

We summarize here the approximations and limitations of the theory described in the previous section. (i) This method relies on the possibility of predicting the closed orbit as a function of the steerer strength, in other words on the validity of Eqs. (5). In a real accelerator the presence of nonlinearities will certainly add a correction to Eqs. (5). The effect of this correction is minimized by keeping the deformation of the closed orbit small so that the nonlinearities affect minimally Eqs. (5). The second order correction to Eqs. (5) would introduce quadratic terms into the equations for ${ }_{x} Q_{t}^{x},{ }_{x} Q_{t}^{y}$ of Eqs. (20), which would then be found as a 4th order correction (in $\theta$ ) in the expression for ${ }_{x} Q_{t i}^{x x},{ }_{x} Q_{t i}^{y y},{ }_{x} Q_{t i}^{x y}$. The knowledge of the orbit response matrices $M^{x}, M^{y}$ is also necessary and it can be acquired via experimental ORM techniques. At the same time it is mandatory to keep the tune sufficiently far from any low order resonance. (ii) We require that the accelerator has horizontal and vertical planes decoupled, which again implies the validity of Eqs. (5). We therefore require $J_{1 l} \sim$ 0 . We also require that gradient errors in the machine-not related to the feed-down - are smaller than the sextupolar and octupolar errors, i.e. $\left|K_{1 l}\right| \ll\left|K_{2 l}\right|,\left|J_{2 l}\right|,\left|K_{3 l}\right|,\left|J_{3 l}\right|$. Note that it would be possible to reformulate this theory in the presence of machine linear coupling at the expense of more complexity. (iii) The beam should be kicked with small angles. This is required to eliminate the transverse dependence of the detuning on high order components. The beam dynamics is then governed by Eqs. (12). When the kick applied on the beam is too strong, the beam oscillation is too large and a different approach is needed in order to include the high order dependences. The methods described in Ref. [19] should be applied for constructing a nonperturbative theory. (iv) The measurements of the tunes have to be sufficiently precise in order to retrieve the terms ${ }_{x} Q_{t}^{x},{ }_{x} Q_{t}^{y},{ }_{x} Q_{t t}^{x x},{ }_{x} Q_{t t}^{y y},{ }_{x} Q_{t t}^{x y}$. From Eqs. (21) we notice that the terms ${ }_{x} Q_{t t}^{x x},{ }_{x} Q_{t t}^{y y},{ }_{x} Q_{t t}^{x y}$ are divided by $\bar{\theta}_{t}^{2}$, which have to be small to fulfill the requirements discussed in (i). Therefore the precision of the tune measurements should scale as $\bar{\theta}_{t}$ to maintain the relative accuracy of each term of the nonlinear tune response matrix. This limits the application of this method up to octupolar errors. For retrieving decapolar errors the precision in measuring the tune should scale as $\bar{\theta}_{t}^{2}$, which is more difficult to determine experimentally. (v) The unavoidable errors in determining ${ }_{x} Q_{t}^{x}$, ${ }_{x} Q_{t}^{y},{ }_{x} Q_{t t}^{x x},{ }_{x} Q_{t t}^{y y},{ }_{x} Q_{t t}^{x y}$ are propagated to the reconstructed strength of nonlinearities $K_{n, l}, J_{n, l}$ according to the characteristics of the orbit response matrices $M^{x}, M^{y}$. Therefore this method will be affected by the possible presence of "small numbers" in the matrices $M^{x}, M^{y}$.

Note that most of these restrictions are fulfilled, if the closed orbit is weakly deformed and if the strength of the 
nonlinear errors is small. We show this in a numerical example.

\section{A. Example on SIS18}

As a first step in validating this theoretical model, we perform a numerical experiment using the SIS18 synchrotron lattice. For the purpose of this example we consider the SIS18 with 12 steerers (horizontal and vertical as discussed in the theory) located at the end of each superperiod. To the linear accelerator we add 12 different nonlinear errors, each of them formed by a thin sextupole and octupole, simultaneously applied to the lattice, located in each superperiod before the quadrupole triplet (see Fig. 2).

We then implemented this accelerator modeling in the MICROMAP library [20]. We set the machine tune at $Q_{x}=$ 4.31, $Q_{y}=3.28$ away from the linear coupling resonance as well as from the $2 \mathrm{nd}, 3 \mathrm{rd}$, and 4 th order resonances. We applied then the method described in Sec. II for reconstructing these nonlinear errors. The closed orbit was deformed via each of the 12 steerers. For each setting of a steerer, we took one particle centered in the deformed closed orbit and induced small betatron oscillations by shifting it along $x$ of $0.1 \mathrm{~mm}$ and along $y$ of $0.3 \mathrm{~mm}$. By taking the $x$ coordinate of the particle over 2048 turns, we compute via a fast-Fourier-transform (FFT) method [21] the induced tuneshift. The strategy described in Sec. II E was then applied to numerically compute ${ }_{x} Q_{t}^{x},{ }_{x} Q_{t}^{y},{ }_{x} Q_{t t}^{x x}$, ${ }_{x} Q_{t t}^{y y},{ }_{x} Q_{t t}^{x y}$. We then solved Eqs. (20) as follows: from ${ }_{x} Q_{t}^{x}$, ${ }_{x} Q_{t}^{y}$ we find $K_{2 l}, J_{2 l}$. We then use these values in the last three equations so that we directly find $K_{3 l}, J_{3 l}$. In Fig. 3 we show the results obtained by applying the NTRM method:

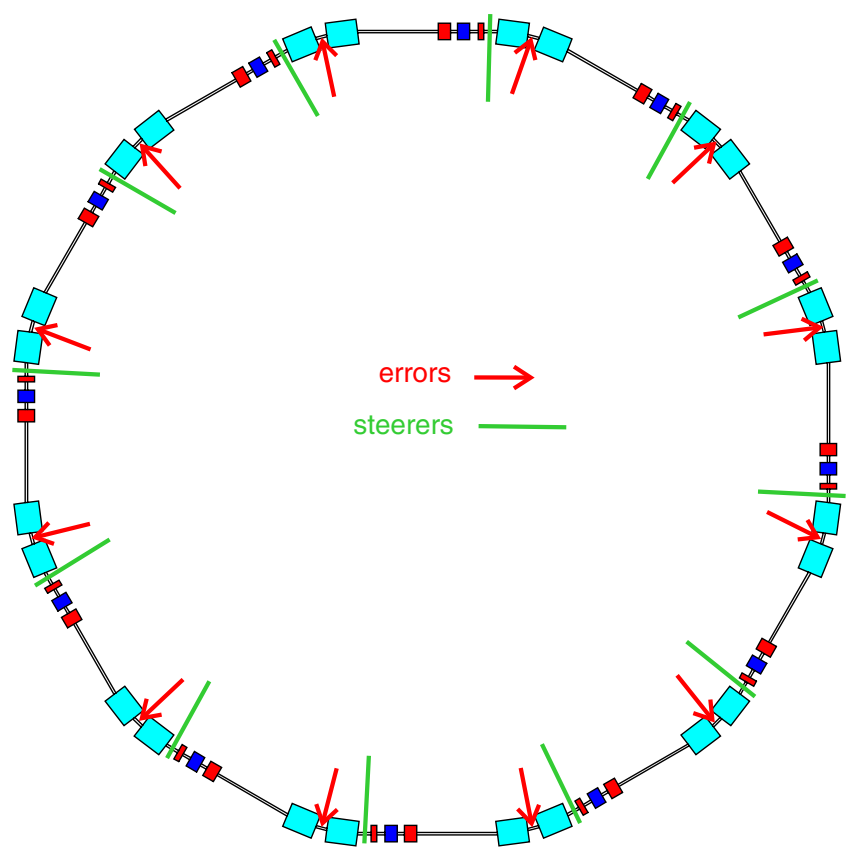

FIG. 2. (Color) SIS18 layout and steerers and nonlinear errors used in the validation simulations. in Fig. 3(a) empty red squared markers give the strength of the reconstructed normal sextupolar errors, and in black the set errors.

With the triangular markers we summarize the results for the skew components. All the results in Fig. 3(a) show the high accuracy of the sextupolar error reconstruction. In Fig. 3(b) are shown the octupolar errors and the reconstructed values [the notations are the same as for Fig. 3(a)]. We here add also the second, redundant, solution for $K_{3 l}$ with green empty squared markers, relative to ${ }_{x} Q_{t t}^{y y}$. As both numerical solutions are practically overlapping, in the
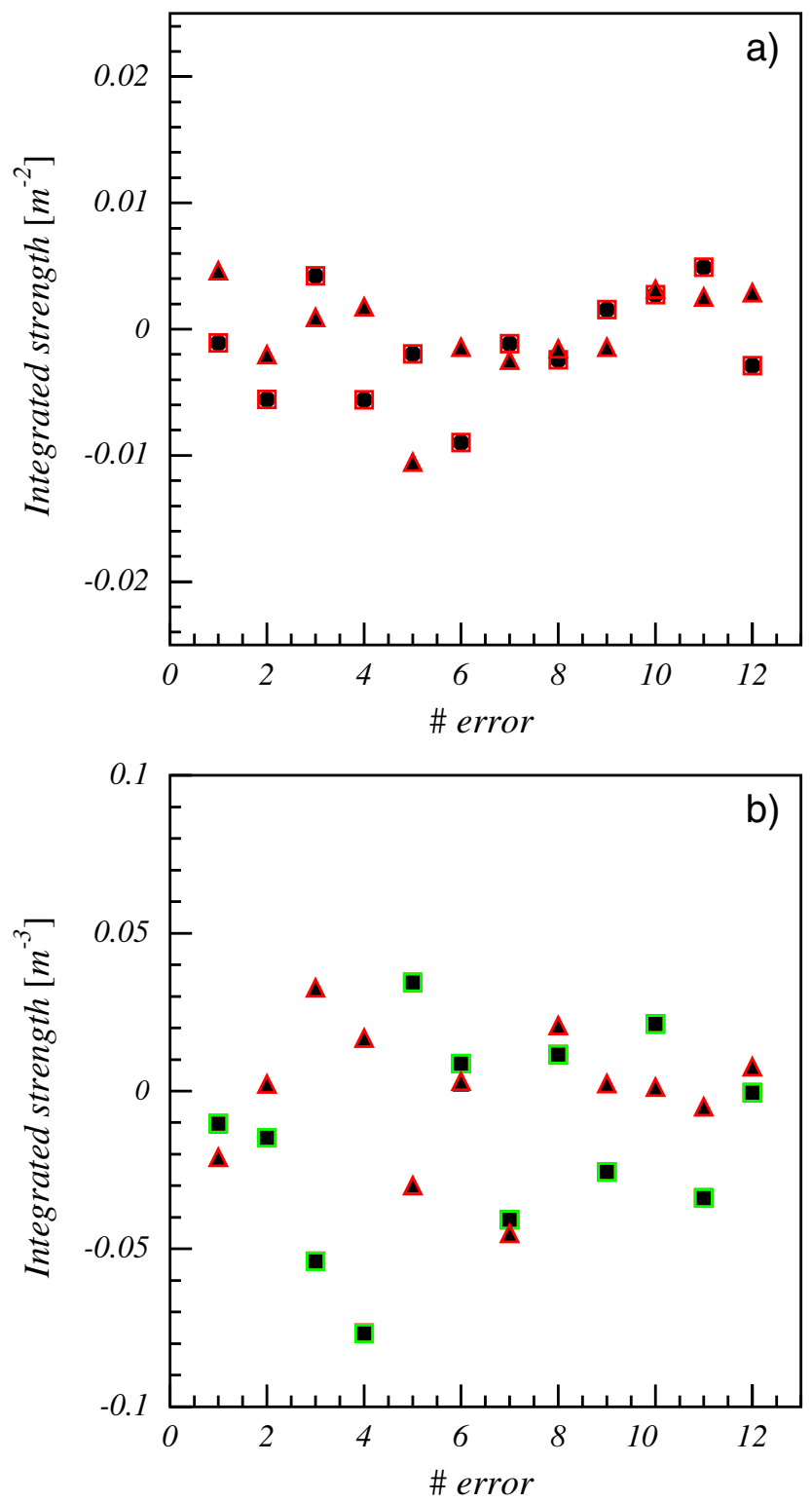

FIG. 3. (Color) Comparison of the reconstructed errors (red markers) with the error set in the SIS18 (black markers). The squared markers refer to normal components, while the triangular markers refer to skew component. In (a) the comparison is shown for sextupolar errors, in (b) for octupolar errors (both solutions for $K_{3 l}$ are plot: red and green squared empty markers). 
picture only the green squared markers are visible. The reconstruction of the octupolar errors largely loses its accuracy if the second order terms resulting from the sextupolar component $\tilde{\mathcal{K}}_{l q}^{x}, \tilde{\mathcal{J}}_{l q}^{x}$ are not included in Eqs. (20).

\section{B. Robustness of the method for realistic beams}

The theory discussed in Sec. II has been developed for a single particle and assumes that the measurement of the tune can be arbitrarily precise. However, in real measurements the beam exhibits a finite emittance and the tunes are measured by kicking the beam with a finite amplitude sometimes far from a perturbative level. In realistic conditions the application of the NTRM method might introduce an error in the evaluation of the experimental tuneshift $\Delta Q_{x, \text { eff }}$. We therefore study the influence of the effect of the finite beam size and several kicked beam amplitudes on the evaluation of the tune. For this study we take the model of the SIS18 synchrotron presently running at GSI, excite all the 12 sextupoles for chromatic correction, and measure the tunes over 4096 turns for several beams of varying emittance and for several amplitudes of the initial kick equally applied in both planes. In
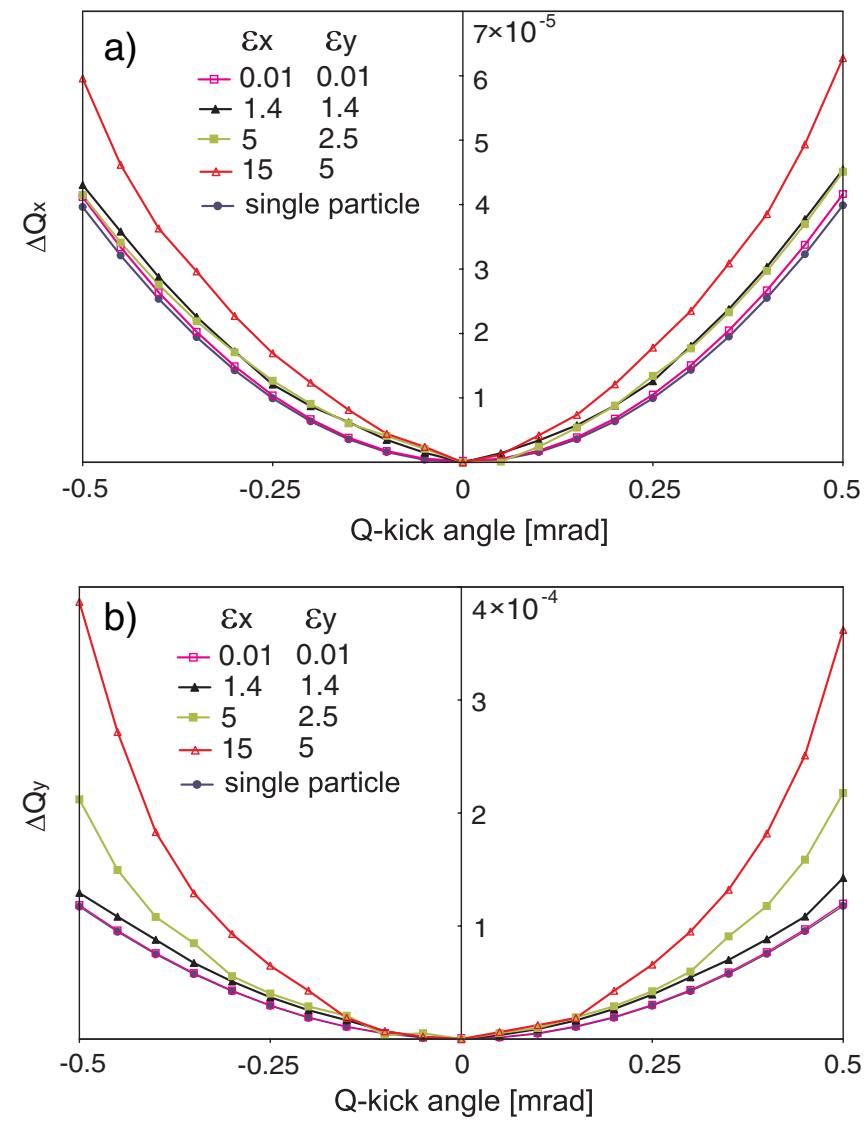

FIG. 4. (Color) Simulation dependence of the nonlinear tune in the horizontal plane (a) and vertical plane (b) as a function of the amplitude of the kicked beam and its emittances. this simulation to distinguish the detuning stemming purely from amplitude effects from the deformed closed orbit effects [described in Eqs. (18) and (19)], we do not deform the closed orbit, but only vary the kick amplitude and beam emittance size.

The multiparticle simulations were performed with 1000 macroparticles. In Fig. 4 we show that the discrepancy of the tune found from a single particle with respect to the tune of a beam with maximum emittances $\epsilon_{x}, \epsilon_{y}<$ $15 \mathrm{~mm}$ mrad is less than $5 \times 10^{-5}$ in the horizontal and vertical planes for a kick amplitude of $\sim 0.1 \mathrm{mrad}$. Note that no octupoles are present in this simulation, and the curvature of tune curves only stems from the large kick amplitude effect.

\section{EXPERIMENTAL VALIDATION ON SIS18}

For the purpose of verifying the effectiveness of the NTRM method, we performed a proof of principle experiment in the SIS18 synchrotron. The testing method is based on the capability of the NTRM method to retrieve a controlled nonlinear error present in the SIS18. We are limited by the fact that the SIS18 is only provided with sextupole magnets for chromatic corrections and slow extraction and no other higher order magnets as octupoles are available. For measuring the tune response, a small emittance beam is created and kicked for inducing transverse betatron oscillations. In order to prevent fast beam oscillation decoherence, the machine chromaticity was corrected. This causes additional 3rd order resonances and quadratic nonlinear components which add to the quadratic nonlinear components preexisting in the SIS18. Therefore setting any controlled sextupolar error as probing nonlinearity would simply be added to the existing known and unknown nonlinearities hindering the testing method. This can be solved by noting that, following Eq. (18), the tune response to the orbit deformation is linear in the error strength for 3 rd order errors [see the terms ${ }_{x} Q_{t}^{x},{ }_{x} Q_{t}^{y}$ in Eqs. (20)]. We take advantage of this dependence to perform the proof of principle experiment as follows: first, we measure the tune response for the machine set for normal operation with chromaticity components (we will refer to this setting as $S 0$ ), then we add on two sextupoles for chromatic correction a small extra probing strength and remeasure the tune response to the same deformation of closed orbit. By subtracting the two tune response curves the resulting differential tune response depends solely from the extra probing error added on the sextupoles. As the probing errors are folded linearly into the terms ${ }_{x} Q_{1}^{x}$, and ${ }_{x} Q_{2}^{x}$, the experimental task is measuring the differential tune response and obtaining ${ }_{x} Q_{1}^{x}$, and ${ }_{x} Q_{2}^{x}$.

\section{A. First proof of principle experiment}

Following the outlined procedure, we have considered the case of two normal probing sextupolar errors to be reconstructed by deforming the orbit by means of two 
TABLE II. Elements of SIS18 used for the first proof of principle experiment.

\begin{tabular}{ccc}
\hline \hline & $t$ & $l$ \\
\hline 1 & S10MU1A & S03KS1C \\
2 & S11MU1A & S11KS1C \\
\hline \hline
\end{tabular}

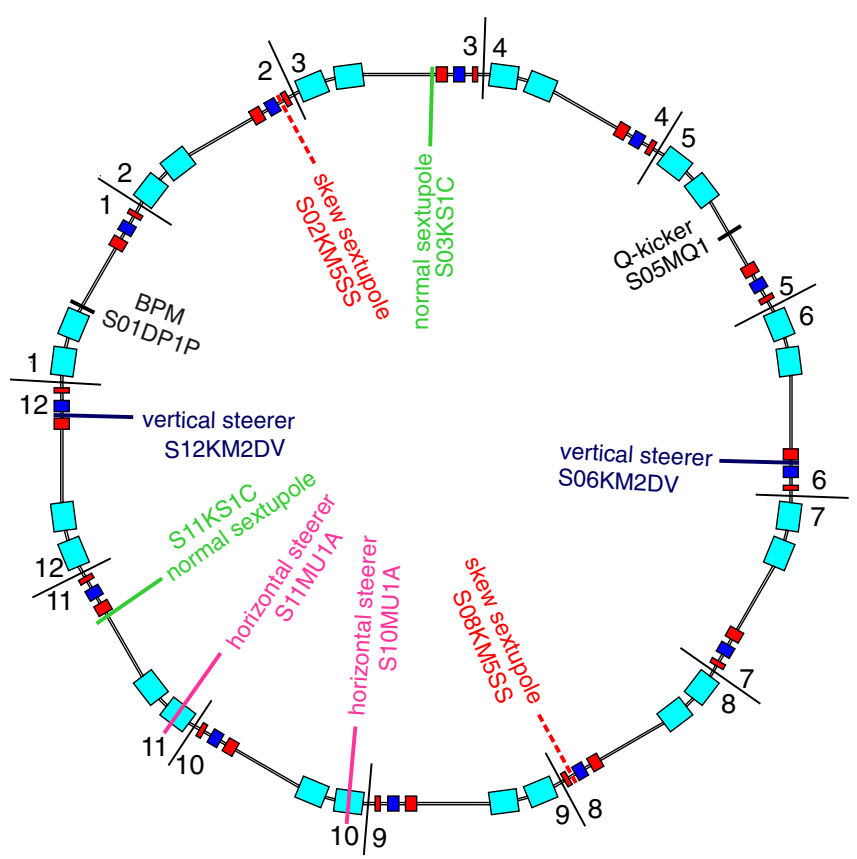

FIG. 5. (Color) SIS18 layout and magnet and steerers used for the proof of principle experiment.

horizontal steerers: as we excite normal probing errors, only horizontal deformation of the closed orbit can reveal them (the terms ${ }_{x} Q_{t}^{y},{ }_{y} Q_{t}^{y}$ are absent). According to the notations of Sec. II we have $N_{l}=2$ and $N_{t}=2$. The elements of SIS18, steerers and sextupoles, associated to the indexes $t$ and $l$ are reported in Table II, and their location in the ring can be seen in Fig. 5.

In the experiment for correcting the chromaticity we have activated the sextupole family S\#KS1C with $K_{2}=$
$-0.2162 \mathrm{~m}^{-2}$, and the family S\#KS3C with $K_{2}=$ $0.4004 \mathrm{~m}^{-2}$ (here $\#=1,3,5,7,9,11$ ). A beam of ${ }^{40} \mathrm{Ar}^{18+}$ was injected into the SIS18 at $11.2 \mathrm{MeV} / \mathrm{u}$ and accelerated at $416.54 \mathrm{MeV} / \mathrm{u}$. The unnormalized beam emittances are estimated to be $\epsilon_{x}=\epsilon_{y} \simeq 1.4 \mathrm{~mm} \mathrm{mrad}$. At this energy we excited a beam oscillation using the fast kicker by a kick of $\sim 0.15 \mathrm{mrad}$ in each plane. The signal was then measured by a beam position monitor.

A FFT analysis of the stored signal over 2048 turns yields the tune response function to the orbit deformation. The tunes of the machine are thus found to be $Q_{x 0}=$ 4.3035, $Q_{y 0}=3.2686$. For completeness, we repeated the measurement for several probing error strengths $\Delta K_{2}$, whose values are reported in the third column of Table III. In Figs. 6(a) and 6(c) are shown the tunes measured as a function of the strength and of the steering angle for the several sets of probing errors of Table III.

In Figs. 6(b) and 6(d) we show the difference tune response curves. As expected these curves are linear and their slopes directly give the quantities ${ }_{x} Q_{1}^{x}$, and ${ }_{x} Q_{2}^{x}$.

Using these quantities, and by inverting Eqs. (20), we retrieve the strength of the probing sextupolar errors. These results are summarized in the fifth column of Table III. For completeness we compute the same quantities via computer simulations and report these results in the fourth column of Table III. In the last column of Table III we show the relative errors of the reconstructed probing errors obtained in the experiment with respect to the originally set value ( 3 rd column). These results fully validate the NTRM as we can retrieve the probing errors with a good accuracy.

\section{B. Second proof of principle experiment}

For the second experimental validation test, we excited two probing errors in skew sextupoles as summarized in Table IV. As the 3rd order errors are now skew, a tune response is expected for vertical closed orbit deformation. For this measurement we measured the tune in the vertical plane to test the NTRM by measuring ${ }_{y} Q_{t}^{y}$.

The experimental setup is the same as for the first experiment, and the strength of the skew probing errors, limited by hardware constraints, is shown in Table V. In

TABLE III. Additional strength applied in the normal sextupoles and retrieved values via NTRM.

\begin{tabular}{llrrrr}
\hline \hline Setting & $l$ & $\Delta K_{2}\left[\mathrm{~m}^{-2}\right] \times 10^{-2}$ & Simulation $\left[\mathrm{m}^{-2}\right] \times 10^{-2}$ & Experiment $\left[\mathrm{m}^{-2}\right] \times 10^{-2}$ & Relative error $\%$ \\
\hline S1 & 1 & -2 & -1.999 & -1.797 & -10.5 \\
& 2 & 1 & 1.001 & 1.018 & -1.8 \\
\hline S2 & 1 & -4 & -3.998 & -4.133 & 3.3 \\
& 2 & 2 & 2.002 & 1.546 & -22.7 \\
\hline S3 & 1 & -8 & -7.995 & -7.609 & -4.9 \\
& 2 & 4 & 4.007 & 3.902 & -2.5 \\
\hline S4 & 1 & 5 & 5.008 & 4.971 & -0.6 \\
& 2 & -3 & -2.997 & -2.739 & -8.7 \\
\hline \hline
\end{tabular}



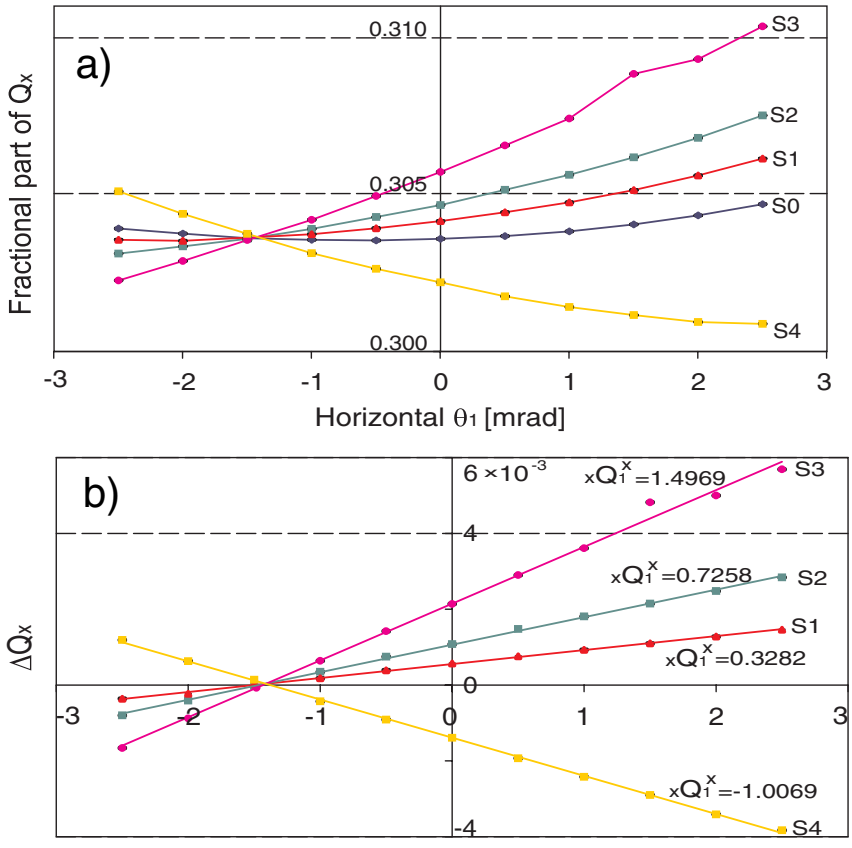

Horizontal $\theta_{1}$ [mrad]
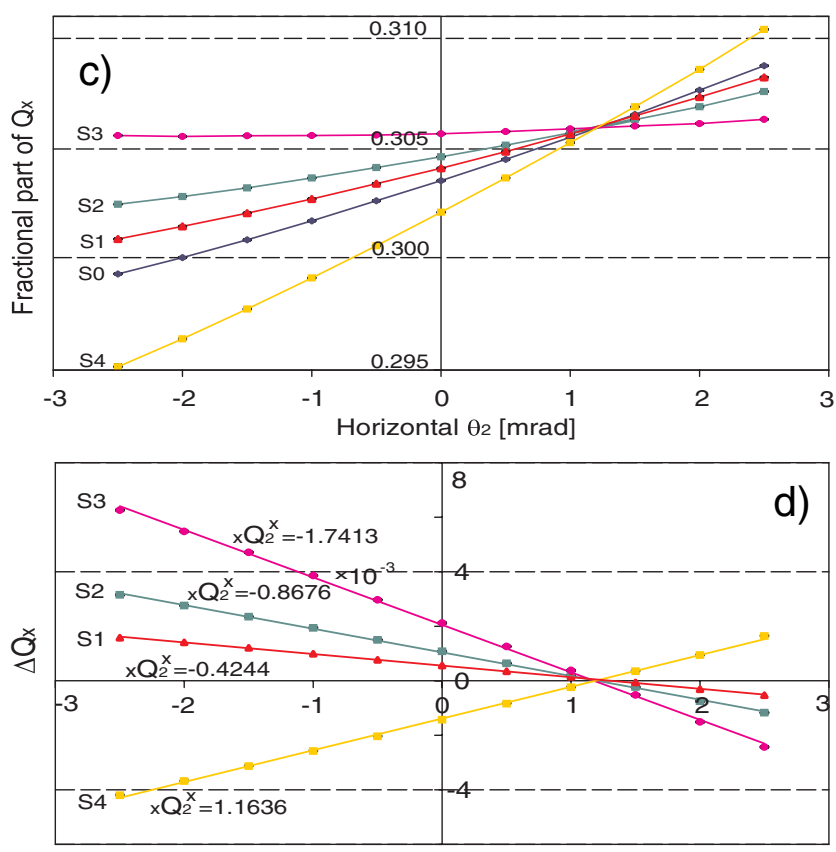

Horizontal $\theta_{2}$ [mrad]

FIG. 6. (Color) Measured tune response for several strengths of the normal error deforming the closed orbit with the first steerer (a) and difference tune response (b). In (c) and (d) the same quantities are obtained varying the second steerer.

TABLE IV. Elements of SIS18 used for the second proof of principle experiment.

\begin{tabular}{llc}
\hline \hline & $t$ & $l$ \\
\hline 1 & S12KM2DV & S02KM5SS \\
2 & S06KM2DV & S08KM5SS \\
\hline \hline
\end{tabular}

Figs. 7(a) and 7(c) we show the tune response curves for these two probing errors. The parabolic behavior can be explained by Eqs. (18) and (20). In fact, we performed the measurements by keeping the chromaticity corrected in order to avoid a fast decoherence of the kicked beam. This is obtained by powering 12 sextupoles, and as the
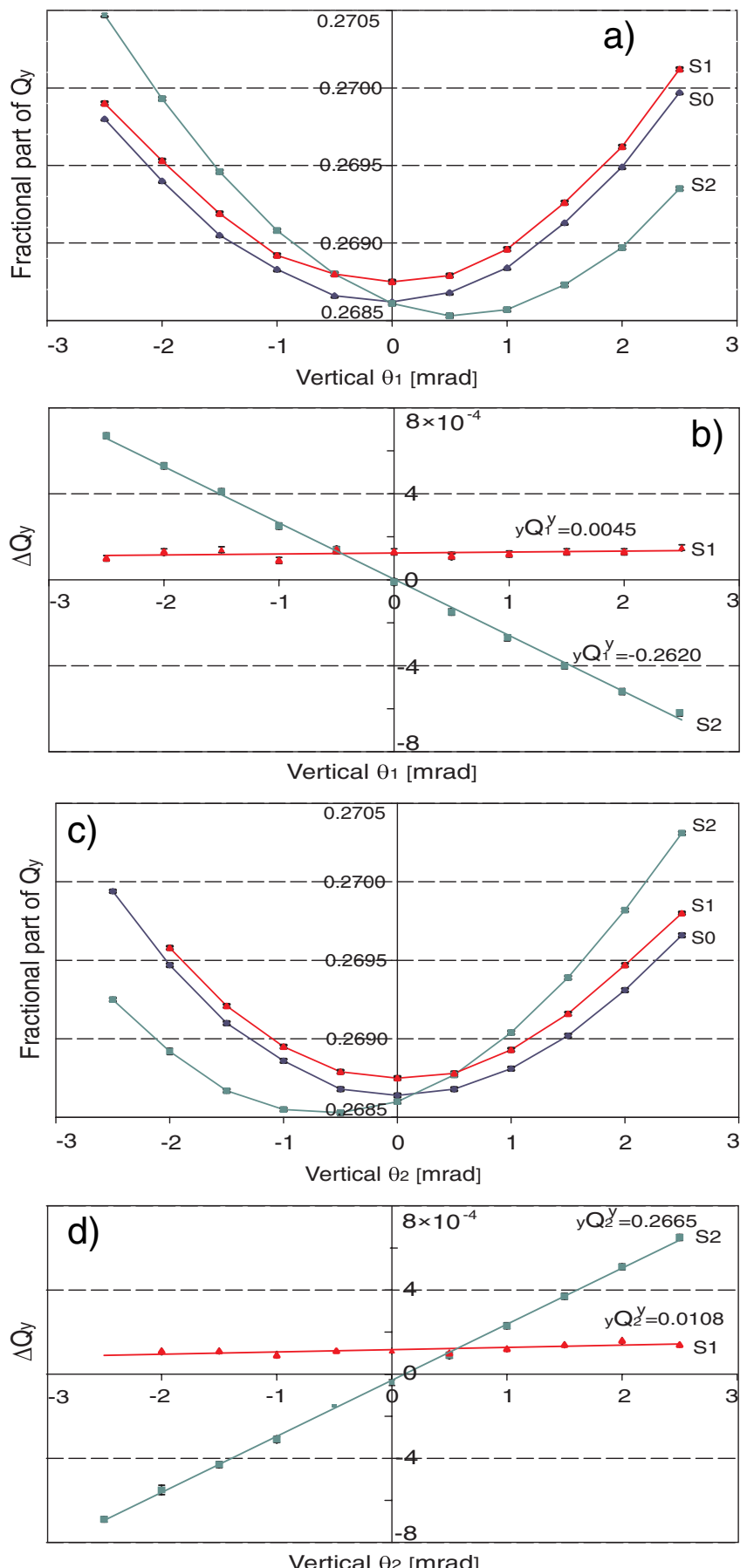

FIG. 7. (Color) Measured tune response for several strengths of the skew error deforming the closed orbit with the first steerer (a) and differential tune response (b). In (c) and (d) the same quantities were obtained varying the second steerer. 
TABLE V. Additional strength applied in the skew sextupoles and retrieved value via NTRM.

\begin{tabular}{llcccc}
\hline \hline Setting & $l$ & $\Delta J_{2}\left[\mathrm{~m}^{-2}\right] \times 10^{-3}$ & Simulation $\left[\mathrm{m}^{-2}\right] \times 10^{-3}$ & Experiment $\left[\mathrm{m}^{-2}\right] \times 10^{-3}$ & Relative error $\%$ \\
\hline S1 & 1 & 8.32 & 8.35 & 7.13 & -14.6 \\
& 2 & 8.32 & 8.35 & 7.29 & -12.7 \\
\hline S2 & 1 & 8.32 & 8.35 & 8.76 & 5.2 \\
& 2 & -8.32 & -8.35 & -4.52 & -45.6 \\
\hline \hline
\end{tabular}

closed orbit is deformed vertically in this measurement, the normal sextupoles feed-down a linear coupling error as shown in the example of Eqs. (15) and (16). In terms of the equations of NTRM, Eqs. (18) and (20), this is equivalent to the excitation of the terms ${ }_{y} Q_{t i}^{x x}$, and ${ }_{y} Q_{t i}^{y y}$ even if natural octupolar errors are absent [the terms ${ }_{y} Q_{t i}^{x x}$, and ${ }_{y} Q_{t i}^{y y}$ are not explicitly written in Eqs. (20), but their form is the same provided that $\tilde{\mathcal{K}}_{l}^{x}, \tilde{\mathcal{K}}_{l q}^{x}, \tilde{\mathcal{J}}_{l q}^{x}$ are substituted with $\tilde{\mathcal{K}}_{l}^{y}$, $\left.\tilde{\mathcal{K}}_{l q}^{y}, \tilde{\mathcal{J}}_{l q}^{y}\right]$. Note that from Eqs. (20) the terms ${ }_{x} Q_{t i}^{x x}$ are excited also in the first proof of principle experiment, but from Figs. 6(a) and 6(c) we see only a very weak trace of a parabolic pattern, hence we conclude that the terms $K_{2 l} K_{2 q} \tilde{\mathcal{K}}_{l q}^{x}$ as well as $K_{3 l}$ are small (not surprisingly as in SIS18 there are not octupoles and error octupoles are typically small). Contrary to the small effect produced by the normal quadrupole component errors, the effect of the induced linear coupling is stronger: we checked this by inspecting the matrix $\tilde{\mathcal{J}}_{l q}^{y}$, which has elements larger than the elements of $\tilde{\mathcal{K}}_{l q}^{y}$. This property of the lattice is the source of the tune response found here. The explicit form of the $\tilde{\mathcal{K}}_{l}^{x}, \tilde{\mathcal{K}}_{l q}^{x}, \tilde{\mathcal{J}}_{l q}^{x}$, and $\tilde{\mathcal{K}}_{l}^{y}, \tilde{\mathcal{K}}_{l q}^{y}, \tilde{\mathcal{J}}_{l q}^{y}$ is given in the Appendix. Note, however, that the difference tune response curves in Figs. 7(b) and 7(d) exhibit only a linear pattern as expected. In fact, the difference of the tunes depends only on the extra probing errors, which are two small skew sextupoles of strength as in Table V. The application of NTRM gives the results summarized in Table V (fifth column). We notice that the reconstruction is less accurate here than in the first experiment, but the probing errors used are very small (because of hardware limitations). In the setting $S 2$ we find that the reconstruction gives $45 \%$ error with respect to the original set value for one of the two skew sextupoles. It cannot be excluded that a larger than assumed discrepancy between lattice model and real lattice is responsible for this large error. Further investigation of this issue is needed.

\section{CONCLUSION AND OUTLOOK}

In this paper we have presented a method for measuring nonlinear components in a circular accelerator. The theoretical basis is discussed and a proof of principle experiment is performed. Our numerical examples show that the reconstruction of sextupolar and octupolar errors is sufficiently accurate to be used in experiments. In the experi- mental tests we found that the reconstruction accuracy is in most of the cases better than $10 \%$ for normal sextupolar errors, and better than $15 \%$ for skew sextupolar errors. The discussion on the origin of some large error is left to future work, where we will repeat these measurements in better experimental condition. The good reconstruction of the nonlinear errors in the majority of the experimental tests, the good results in numerical examples, as well as the simple foundation of the theoretical approach allow us to conclude that this method can be applied to large accelerators provided that the linear optics has been studied and modeled before. Clearly, the NTRM method is affected by the accuracy of the tune measurements, therefore it is unlikely that this method can be applied to errors of order higher than the 4th, as the tune accuracy required would exceed $10^{-6}$. It is planned to further test this method at GSI for a larger set of controlled probing errors and eventually apply it to the measurement of the inherent random nonlinear errors.

\section{ACKNOWLEDGMENTS}

The authors are indebted to W. Bayer for his support in the measurements; to P. Spiller for the coordination of the machine experiments; and to U. Blell and M. Petryk for the assistance with the Q-kicker.

\section{APPENDIX A}

In order to predict the oscillation frequencies of a particle around the closed orbit, the one-turn map $\mathbf{M}_{T}$ has to be found. In the particular circumstance of a lattice with deformed closed orbit, the linear lattice will be composed by a sequence of $4 \times 4$ linear (decoupled) transfer maps $\mathbf{M}_{l}^{*}$, where

$$
\mathbf{M}_{l}^{*}=\left(\begin{array}{cc}
\mathbf{M}_{x l}^{*} & 0 \\
0 & \mathbf{M}_{y l}^{*}
\end{array}\right)
$$

alternated by feed-down induced linear errors $\Delta_{l}$. The $2 \times$ 2 matrices $\mathbf{M}_{x l}^{*}, \mathbf{M}_{y l}^{*}$, are the transport maps between the linear errors $\boldsymbol{\Delta}_{l}$. The index $\boldsymbol{l}$ is enumerating the sequence of matrices and is not meant as matrix component (hence the symbol is in bold). The most general form of the $l$ th linear error can be written in matrix form as

$$
\Delta_{l}=\mathbf{I}+\mathbf{E}_{l},
$$

where $\mathbf{I}$ is the $4 \times 4$ identity matrix and 


$$
\mathbf{E}_{l}=\left(\begin{array}{cc}
-\mathbf{K}_{l} & \mathbf{J}_{l} \\
\mathbf{J}_{l} & \mathbf{K}_{l}
\end{array}\right)
$$

with $\mathbf{K}_{l}, \mathbf{J}_{l}$ the $2 \times 2$ matrices

$$
\mathbf{K}_{l}=\left(\begin{array}{cc}
0 & 0 \\
\tilde{k}_{l} & 0
\end{array}\right), \quad \mathbf{J}_{l}=\left(\begin{array}{cc}
0 & 0 \\
\tilde{j}_{l} & 0
\end{array}\right) .
$$

The terms $\tilde{k}_{l}, \tilde{j}_{l}$ are the strength of the feed-down induced linear errors obtained in Eqs. (14). The index $l$ refers to the location of the nonlinear errors. From the point of view of the linear optics the accelerator lattice, including the feed-down induced linear errors, is now composed of the sequence of linear objects $\mathbf{M}_{1}^{*}, \boldsymbol{\Delta}_{1}, \mathbf{M}_{2}^{*}, \boldsymbol{\Delta}_{2}, \mathbf{M}_{3}^{*}$, $\boldsymbol{\Delta}_{3}, \ldots, \mathbf{M}_{N_{l}}^{*}, \boldsymbol{\Delta}_{N_{l}}$, and the one-turn map is then obtained as

$$
\mathbf{M}_{T}=\prod_{l=1}^{N_{l}} \Delta_{l} \mathbf{M}_{l}^{*}=\Delta_{N_{l}} \mathbf{M}_{N_{l}}^{*} \ldots \Delta_{2} \mathbf{M}_{2}^{*} \Delta_{1} \mathbf{M}_{1}^{*}
$$

By substituting Eq. (A2) into Eq. (A3) we obtain an expansion of the one-turn map $\mathbf{M}_{T}$, namely

$$
\mathbf{M}_{\boldsymbol{T}}=\mathbf{M}_{\boldsymbol{T}}^{*}+\boldsymbol{\delta}^{(\mathbf{1})} \mathbf{M}^{*}+\boldsymbol{\delta}^{(\mathbf{2})} \mathbf{M}^{*}+O(3),
$$

where

$$
\begin{aligned}
\boldsymbol{\delta}^{(\mathbf{1})} \mathbf{M}^{*} & =\sum_{l=1}^{N_{l}} \check{\mathbf{M}}_{l} \mathbf{E}_{l} \mathbf{M}_{l} \\
\boldsymbol{\delta}^{(\mathbf{2})} \mathbf{M}^{*} & =\sum_{l>j} \check{\mathbf{M}}_{l} \mathbf{E}_{l} \mathbf{M}_{[l j]} \mathbf{E}_{j} \mathbf{M}_{\boldsymbol{j}}
\end{aligned}
$$

In Eq. (A4) the matrix $\mathbf{M}_{T}^{*}$ is the $4 \times 4$ unperturbed oneturn map $\mathbf{M}_{\boldsymbol{T}}^{*}=\prod_{l=1}^{N_{l}} \mathbf{M}_{l}^{*}$ which we can write as

$$
\mathbf{M}_{T}^{*}=\left(\begin{array}{cc}
\mathbf{M}_{\boldsymbol{x}} & 0 \\
0 & \mathbf{M}_{\boldsymbol{y}}
\end{array}\right)
$$

where $\mathbf{M}_{\boldsymbol{x}}=\prod_{l=1}^{N_{l}} \mathbf{M}_{\boldsymbol{x} \boldsymbol{l}}^{*}, \mathbf{M}_{\boldsymbol{y}}=\prod_{l=1}^{N_{l}} \mathbf{M}_{\boldsymbol{y} \boldsymbol{l}}^{*}$. In Eqs. (A5) we also define $\mathbf{M}_{\boldsymbol{l}}=\prod_{i=1}^{l} \mathbf{M}_{i}^{*}$, and $\check{\mathbf{M}}_{\boldsymbol{l}}=\prod_{i=l+1}^{N_{l}} \mathbf{M}_{i}^{*}$ as the two transport matrices between the error $\mathbf{E}_{l}$ and therefore $\check{\mathbf{M}}_{l} \mathbf{M}_{l}=\mathbf{M}_{T}^{*} . \quad$ Consistently $\quad \mathbf{M}_{x l}=\prod_{i=1}^{l} \mathbf{M}_{x i}^{*}, \quad \check{\mathbf{M}}_{x l}=$ $\prod_{i=l+1}^{N_{l}} \mathbf{M}_{\boldsymbol{x} \boldsymbol{i}}^{*}$, and $\mathbf{M}_{\boldsymbol{y} \boldsymbol{l}}=\prod_{i=1}^{l} \mathbf{M}_{\boldsymbol{y} \boldsymbol{i}}^{*}, \quad \mathbf{M}_{\boldsymbol{y} \boldsymbol{l}}=\prod_{i=l+1}^{N_{l}} \mathbf{M}_{\boldsymbol{y} \boldsymbol{i}}^{*}$. The matrix $\mathbf{M}_{[l j]}$ which describe the relation between the linear error $\mathbf{E}_{\boldsymbol{l}}$, and $\mathbf{E}_{j}$ is defined as $\mathbf{M}_{[l j]}=\prod_{i=j+1}^{l} \mathbf{M}_{i}^{*}$. Again $\mathbf{M}_{x[l j]}=\prod_{i=j+1}^{l} \mathbf{M}_{x i}^{*}$, and $\mathbf{M}_{y[l j]}=\prod_{i=j+1}^{l} \mathbf{M}_{\boldsymbol{y} \boldsymbol{i}}^{*}$. The symplectic one-turn map, written in blocks, reads

$$
\mathbf{M}_{T}=\left(\begin{array}{ll}
\mathbf{M} & \mathbf{n} \\
\mathbf{m} & \mathbf{N}
\end{array}\right)
$$

where

$$
\begin{aligned}
\mathbf{M}= & \mathbf{M}_{x}-\sum_{l=1}^{N_{l}} \check{\mathbf{M}}_{x l} \mathbf{K}_{l} \mathbf{M}_{x l}+\sum_{l>j}\left[\check{\mathbf{M}}_{x l} \mathbf{K}_{l} \mathbf{M}_{x[l j]} \mathbf{K}_{j} \mathbf{M}_{x j}\right. \\
& \left.+\check{\mathbf{M}}_{x l} \mathbf{J}_{l} \mathbf{M}_{\boldsymbol{y}[l j]} \mathbf{J}_{j} \mathbf{M}_{x j}\right]+O(3), \\
\mathbf{n}= & \sum_{l=1}^{N_{l}} \check{\mathbf{M}}_{x l} \mathbf{J}_{l} \mathbf{M}_{y l}-\sum_{l>j}\left[\check{\mathbf{M}}_{x l} \mathbf{K}_{l} \mathbf{M}_{x[l j]} \mathbf{J}_{j} \mathbf{M}_{y j}\right. \\
& \left.-\check{\mathbf{M}}_{x l} \mathbf{J}_{l} \mathbf{M}_{y[l j]} \mathbf{K}_{j} \mathbf{M}_{y j}\right]+O(3), \\
\mathbf{m}= & \sum_{l=1}^{N_{l}} \check{\mathbf{M}}_{y l} \mathbf{J}_{l} \mathbf{M}_{x l}-\sum_{l>j}\left[\check{\mathbf{M}}{ }_{y l} \mathbf{J}_{l} \mathbf{M}_{x[l j]} \mathbf{K}_{j} \mathbf{M}_{x j}\right. \\
& \left.-\check{\mathbf{M}}_{y l} \mathbf{K}_{l} \mathbf{M}_{y[l j]} \mathbf{J}_{j} \mathbf{M}_{x j}\right]+O(3), \\
\mathbf{N}= & \mathbf{M}_{\boldsymbol{y}}+\sum_{l=1}^{N_{l}} \check{\mathbf{M}}_{y l} \mathbf{K}_{l} \mathbf{M}_{y l}+\sum_{l>j}\left[\check{\mathbf{M}}{ }_{y l} \mathbf{J}_{l} \mathbf{M}_{x[l j]} \mathbf{J}_{j} \mathbf{M}_{y j}\right. \\
& \left.+\check{\mathbf{M}}_{y l} \mathbf{K}_{l} \mathbf{M}_{y[l j]} \mathbf{K}_{j} \mathbf{M}_{y j}\right]+O(3) .
\end{aligned}
$$

The frequencies of oscillation of a particle around the deformed closed orbit are obtained by finding the normal modes of the one-turn map Eq. (A7), which are implicitly given by $\Lambda_{+}=2 \cos \left[2 \pi\left(Q_{x 0}+\Delta Q_{x}\right)\right]$, and $\Lambda_{-}=$ $2 \cos \left[2 \pi\left(Q_{y 0}+\Delta Q_{y}\right)\right]$, where $\Delta Q_{x}, \Delta Q_{y}$ are the tuneshifts induced by linear feed-down in the presence of closed orbit deformation. The quantity $\Lambda_{ \pm}$is given by [22]

$$
\begin{aligned}
2 \Lambda_{ \pm}= & \operatorname{Tr}(\mathbf{M})+\operatorname{Tr}(\mathbf{N}) \\
& \pm \sqrt{[\operatorname{Tr}(\mathbf{M})-\operatorname{Tr}(\mathbf{N})]^{2}+4|\mathbf{m}+\overline{\mathbf{n}}|}
\end{aligned}
$$

where $\overline{\mathbf{n}}=-\mathbf{S n}^{\dagger} \mathbf{S}$. The symbol ${ }^{\dagger}$ is used for the transpose of a matrix and $\mathbf{S}$ is the symplectic matrix

$$
\mathbf{S}=\left(\begin{array}{cc}
0 & 1 \\
-1 & 0
\end{array}\right)
$$

If the natural linear coupling is small such that $4|\mathbf{m}+\overline{\mathbf{n}}|<$ $\operatorname{Tr}(\mathbf{M})-\operatorname{Tr}(\mathbf{N})$, we can expand Eq. (A9) as

$$
\begin{aligned}
& \Lambda_{+}=\operatorname{Tr}(\mathbf{M})+\frac{|\mathbf{m}+\overline{\mathbf{n}}|}{\operatorname{Tr}(\mathbf{M})-\operatorname{Tr}(\mathbf{N})}+O(3), \\
& \Lambda_{-}=\operatorname{Tr}(\mathbf{N})-\frac{|\mathbf{m}+\overline{\mathbf{n}}|}{\operatorname{Tr}(\mathbf{M})-\operatorname{Tr}(\mathbf{N})}+O(3) .
\end{aligned}
$$

The condition of validity of this expansion is obtained by keeping the tunes far from the linear coupling so that $\operatorname{Tr}(\mathbf{M}) \neq \operatorname{Tr}(\mathbf{N})$ and to limit this expansion to a lattice with small nonlinear errors and perturbed by small closed orbit deformations so that $|\mathbf{m}+\overline{\mathbf{n}}|$ is small. Now the traces can be written as 


$$
\begin{aligned}
& \operatorname{Tr}(\mathbf{M})=\operatorname{Tr}\left(\mathbf{M}_{x}\right)-\sum_{l=1}^{N_{l}} \operatorname{Tr}\left(\check{\mathbf{M}}_{x l} \mathbf{K}_{l} \mathbf{M}_{x l}\right) \\
& +\sum_{l>j}\left[\operatorname{Tr}\left(\check{\mathbf{M}}_{x l} \mathbf{K}_{l} \mathbf{M}_{x[l j]} \mathbf{K}_{j} \mathbf{M}_{x j}\right)\right. \\
& \left.+\operatorname{Tr}\left(\check{\mathbf{M}}_{x l} \mathbf{J}_{l} \mathbf{M}_{y[l j]} \mathbf{J}_{j} \mathbf{M}_{x j}\right)\right]+O(3), \\
& \operatorname{Tr}(\mathbf{N})=\operatorname{Tr}\left(\mathbf{M}_{\boldsymbol{y}}\right)+\sum_{l=1}^{N_{l}} \operatorname{Tr}\left(\check{\mathbf{M}}_{\boldsymbol{y} l} \mathbf{K}_{\boldsymbol{l}} \mathbf{M}_{\boldsymbol{y} l}\right) \\
& +\sum_{l>j}\left[\operatorname{Tr}\left(\check{\mathbf{M}}_{y l} \mathbf{J}_{l} \mathbf{M}_{x[l j]} \mathbf{J}_{j} \mathbf{M}_{y j}\right)\right. \\
& \left.+\operatorname{Tr}\left(\check{\mathbf{M}}_{\boldsymbol{y l}} \mathbf{K}_{\boldsymbol{l}} \mathbf{M}_{\boldsymbol{y}[\boldsymbol{l} \boldsymbol{j}]} \mathbf{K}_{\boldsymbol{j}} \mathbf{M}_{\boldsymbol{y} \boldsymbol{j}}\right)\right]+O(3) .
\end{aligned}
$$

It is now straightforward to prove that $\operatorname{Tr}(\mathbf{M})=$ $2 \cos \left(2 \pi Q_{x 0}\right), \operatorname{Tr}(\mathbf{N})=2 \cos \left(2 \pi Q_{y 0}\right)$ and

$$
\begin{aligned}
& \operatorname{Tr}\left(\check{\mathbf{M}}_{x l} \mathbf{K}_{l} \mathbf{M}_{x l}\right)=\sin \left(2 \pi Q_{x 0}\right) \beta_{x l} \tilde{k}_{l}, \\
& \operatorname{Tr}\left(\check{\mathbf{M}}_{y l} \mathbf{K}_{l} \mathbf{M}_{y l}\right)=\sin \left(2 \pi Q_{y 0}\right) \beta_{y l} \tilde{k}_{l},
\end{aligned}
$$

and

$$
\begin{aligned}
& \operatorname{Tr}\left(\check{\mathbf{M}}_{\boldsymbol{x} l} \mathbf{K}_{\boldsymbol{l}} \mathbf{M}_{\boldsymbol{x}[\boldsymbol{l j}]} \mathbf{K}_{j} \mathbf{M}_{\boldsymbol{x} j}\right)=\beta_{x l} \beta_{x j} \mathcal{S}_{x,[l j]} \check{\mathcal{S}}_{x,[l j]} \tilde{k}_{l} \tilde{k}_{j}, \\
& \operatorname{Tr}\left(\check{\mathbf{M}}_{x l} \mathbf{J}_{l} \mathbf{M}_{\boldsymbol{y}[\boldsymbol{l} \boldsymbol{j}]} \mathbf{J}_{\boldsymbol{j}} \mathbf{M}_{\boldsymbol{x} \boldsymbol{j}}\right)=\sqrt{\beta_{x l} \beta_{y l} \beta_{x j} \beta_{y j}} \mathcal{S}_{y,[l j]} \check{\mathcal{S}}_{x,[l j]} \tilde{j}_{l} \tilde{j}_{j}, \\
& \operatorname{Tr}\left(\check{\mathbf{M}}_{\boldsymbol{y l}} \mathbf{J}_{\boldsymbol{l}} \mathbf{M}_{\boldsymbol{x}[\boldsymbol{l} \boldsymbol{j}]} \mathbf{J}_{\boldsymbol{j}} \mathbf{M}_{\boldsymbol{y} \boldsymbol{j}}\right)=\sqrt{\beta_{x l} \beta_{y l} \beta_{x j} \beta_{y j}} \mathcal{S}_{x,[l j]} \check{\mathcal{S}}_{y,[l j]} \tilde{j}_{l} \tilde{j}_{j}, \\
& \operatorname{Tr}\left(\check{\mathbf{M}}_{\boldsymbol{y} l} \mathbf{K}_{\boldsymbol{l}} \mathbf{M}_{\boldsymbol{y}[\boldsymbol{l} \boldsymbol{j}]} \mathbf{K}_{\boldsymbol{j}} \mathbf{M}_{\boldsymbol{y} \boldsymbol{j}}\right)=\beta_{y l} \beta_{y j} \mathcal{S}_{y,[l j]} \check{\mathcal{S}}_{y,[l j]} \tilde{k}_{l} \tilde{k}_{j}, \quad(\mathrm{~A} 13)
\end{aligned}
$$

where for sake of simplicity we define $\mathcal{S}_{x,[l j]}=$ $\sin \left(\Delta \psi_{x,[l j]}\right), \mathcal{S}_{y,[l j]}=\sin \left(\Delta \psi_{y,[l j]}\right), \check{\mathcal{S}}_{x,[l j]}=\sin \left(2 \pi Q_{x 0}-\right.$ $\left.\Delta \psi_{x,[l j]}\right), \check{\mathcal{S}}_{y,[l j]}=\sin \left(2 \pi Q_{y 0}-\Delta \psi_{y,[l j]}\right) ;$ with $\Delta \psi_{x,[l j]}=$ $\psi_{x l}-\psi_{x j}$, and $\Delta \psi_{y,[l j]}=\psi_{y l}-\psi_{y j}$. Here $\psi_{x l}, \psi_{x j}, \psi_{y l}$, $\psi_{y j}$ are the phase advances at $s=s_{l}, s=s_{j}$. By substituting $\Delta \psi_{x,[l j]} \rightarrow\left|\Delta \psi_{x,[l j]}\right|$, and $\Delta \psi_{y,[l j]} \rightarrow\left|\Delta \psi_{y,[l j]}\right|$, we symmetrize $\mathcal{S}_{x,[l j]}, \check{\mathcal{S}}_{x,[l j]}, \mathcal{S}_{y,[l j]}, \check{\mathcal{S}}_{y,[l j]}$ for the exchange of indexes $l$ with $j$, i.e., we find $\mathcal{S}_{x,[l j]}=\mathcal{S}_{x,[j l]}$, and $\check{\mathcal{S}}_{x,[l j]}=$ $\check{\mathcal{S}}_{x,[j l]}$. Therefore we can conveniently drop the condition $l>j$ in Eqs. (A11) and write

$$
\begin{aligned}
\operatorname{Tr}(\mathbf{M})= & 2 \cos \left(2 \pi Q_{x 0}\right)-\sin \left(2 \pi Q_{x 0}\right) \sum_{l=1}^{N_{l}} \beta_{x l} \tilde{k}_{l} \\
& +\frac{1}{2} \sum_{l, j=1}^{N_{l}}\left[\beta_{x l} \beta_{x j} \mathcal{S}_{x,[l j]} \check{\mathcal{S}}_{x,[l j]} \tilde{k}_{l} \tilde{k}_{j}\right. \\
& \left.+\sqrt{\beta_{x l} \beta_{y l} \beta_{x j} \beta_{y j}} \mathcal{S}_{y,[l j]} \check{\mathcal{S}}_{x,[l j]} \tilde{j}_{l} \tilde{j}_{j}\right]+O(3), \\
\operatorname{Tr}(\mathbf{N})= & 2 \cos \left(2 \pi Q_{y 0}\right)+\sin \left(2 \pi Q_{y 0}\right) \sum_{l=1}^{N_{l}} \beta_{y l} \tilde{k}_{l} \\
& +\frac{1}{2} \sum_{l, j=1}^{N_{l}}\left[\sqrt{\beta_{x l} \beta_{y l} \beta_{x j} \beta_{y j}} \mathcal{S}_{x,[l j]} \check{\mathcal{S}}_{y,[l j]} \tilde{j}_{l} \tilde{j}_{j}\right. \\
& \left.+\beta_{y l} \beta_{y j} \mathcal{S}_{y,[l j]} \check{\mathcal{S}}_{y,[l j]} \tilde{k}_{l} \tilde{k}_{j}\right]+O(3) .
\end{aligned}
$$

All these findings are summarized in the following formulas:

$$
\begin{aligned}
\Lambda_{+}= & 2 \cos \left(2 \pi Q_{x 0}\right)-\sin \left(2 \pi Q_{x 0}\right) \sum_{l=1}^{N_{l}} \beta_{x l} \tilde{k}_{l} \\
& +\frac{1}{2} \sum_{l, j=1}^{N_{l}}\left[\beta_{x l} \beta_{x j} \mathcal{S}_{x,[l j]} \check{\mathcal{S}}_{x,[l j]} \tilde{k}_{l} \tilde{k}_{j}\right. \\
& \left.+\sqrt{\beta_{x l} \beta_{y l} \beta_{x j} \beta_{y j}} \mathcal{S}_{y,[l j]} \check{\mathcal{S}}_{x,[l j]} \tilde{j}_{l} \tilde{j}_{j}\right] \\
& +\frac{|\mathbf{m}+\overline{\mathbf{n}}|}{2\left[\cos \left(2 \pi Q_{x 0}\right)-\cos \left(2 \pi Q_{y 0}\right)\right]}+O(3),
\end{aligned}
$$

and

$$
\begin{aligned}
\Lambda_{-}= & 2 \cos \left(2 \pi Q_{y 0}\right)+\sin \left(2 \pi Q_{y 0}\right) \sum_{l=1}^{N_{l}} \beta_{y l} \tilde{k}_{l} \\
& +\frac{1}{2} \sum_{l, j=1}^{N_{l}}\left[\sqrt{\beta_{x l} \beta_{y l} \beta_{x j} \beta_{y j}} \mathcal{S}_{x,[l j]} \check{\mathcal{S}}_{y,[l j]} \tilde{j}_{l} \tilde{j}_{j}\right. \\
& \left.+\beta_{y l} \beta_{y j} \mathcal{S}_{y,[l j]} \check{\mathcal{S}}_{y,[l j]} \tilde{k}_{l} \tilde{k}_{j}\right] \\
& -\frac{|\mathbf{m}+\overline{\mathbf{n}}|}{2\left[\cos \left(2 \pi Q_{x 0}\right)-\cos \left(2 \pi Q_{y 0}\right)\right]}+O(3) .
\end{aligned}
$$

\section{Effect of the induced linear coupling}

We calculate in this section the term $|\mathbf{m}+\overline{\mathbf{n}}|$ present in Eqs. (A10). From Eqs. (A8) we find

$$
\mathbf{n}=\sum_{l=1}^{N_{l}} \check{\mathbf{M}}_{x l} \mathbf{J}_{l} \mathbf{M}_{y l}+O(2), \quad \mathbf{m}=\sum_{l=1}^{N_{l}} \check{\mathbf{M}}_{y l} \mathbf{J}_{l} \mathbf{M}_{x l}+O(2) .
$$

In order to compute the normal modes we first compute $\mathbf{m}+\overline{\mathbf{n}}$, which reads

$$
\mathbf{m}+\overline{\mathbf{n}}=\sum_{l=1}^{N_{l}}\left[\check{\mathbf{M}}_{y l} \mathbf{J}_{l} \mathbf{M}_{x l}-\mathbf{S}\left(\check{\mathbf{M}}_{x l} \mathbf{J}_{l} \mathbf{M}_{y l}\right)^{\dagger} \mathbf{S}\right]+O(2) .
$$

For the first term in the square bracket we use the decomposition

$$
\check{\mathbf{M}}_{y l} \mathbf{J}_{l} \mathbf{M}_{x l}=\mathbf{T}_{y} \check{\mathbf{R}}_{y l} \mathbf{T}_{y l}^{-1} \mathbf{J}_{l} \mathbf{T}_{x l} \mathbf{R}_{x l} \mathbf{T}_{x}^{-1},
$$

with

$$
\begin{aligned}
& \mathbf{T}_{\boldsymbol{x} / \boldsymbol{y}}=\left(\begin{array}{cc}
\sqrt{\beta_{x / y}} & 0 \\
-\frac{\alpha_{x / y}}{\sqrt{\beta_{x / y}}} & \frac{1}{\sqrt{\beta_{x / y}}}
\end{array}\right), \\
& \mathbf{T}_{\boldsymbol{x} l / \boldsymbol{y} \boldsymbol{l}}=\left(\begin{array}{cc}
\sqrt{\beta_{x l / y l}} & 0 \\
-\frac{\alpha_{x l / y l}}{\sqrt{\beta_{x l} / y l}} & \frac{1}{\sqrt{\beta_{x l / y l}}}
\end{array}\right),
\end{aligned}
$$

and $\check{\mathbf{R}}_{y l}$ is a rotation of $2 \pi Q_{y 0}-\psi_{y l} ; \mathbf{R}_{x l}$ is a rotation of $\psi_{x l}$. The term $\mathbf{T}_{\boldsymbol{y} l}^{-1} \mathbf{J}_{l} \mathbf{T}_{x l}$ becomes 


$$
\mathbf{T}_{y l}^{-1} \mathbf{J}_{l} \mathbf{T}_{x l}=\left(\begin{array}{cc}
0 & 0 \\
\sqrt{\beta_{x l} \beta_{y l}} \tilde{j}_{l} & 0
\end{array}\right)=\mathbf{C}_{\boldsymbol{I}} \sqrt{\beta_{x l} \beta_{y l}} \tilde{j}_{l},
$$

with

$$
\mathbf{C}_{\boldsymbol{I}}=\left(\begin{array}{ll}
0 & 0 \\
1 & 0
\end{array}\right)
$$

That is we find

$$
\check{\mathbf{M}}_{y l} \mathbf{J}_{l} \mathbf{M}_{x l}=\mathbf{T}_{\boldsymbol{y}} \check{\mathbf{R}}_{y l} \mathbf{C}_{\boldsymbol{I}} \mathbf{R}_{x l} \mathbf{T}_{x}^{-1} \sqrt{\beta_{x l} \beta_{y l}} \tilde{j}_{l} .
$$

For the second term in the square bracket of Eq. (A18) we make use of the symplecticity of the matrices $\check{\mathbf{M}}_{\boldsymbol{y l}}, \mathbf{M}_{\mathbf{y l}}$. We recall that for a generic $2 \times 2$ matrix $\mathbf{A}$ the symplecticity implies $\mathbf{S A}^{\dagger} \mathbf{S}=-\mathbf{A}^{-1}$; also by direct calculation we find the relation $\mathbf{S J}_{l}^{\dagger} \mathbf{S}=\mathbf{J}_{l}$. By using these properties we find

$$
\mathbf{S}\left(\check{\mathbf{M}}_{x l} \mathbf{J}_{l} \mathbf{M}_{y l}\right)^{\dagger} \mathbf{S}=\mathbf{M}_{y l}^{-1} \mathbf{J}_{l} \check{\mathbf{M}}_{x l}^{-1} .
$$

Following the same technique used for the first term in the square brackets of Eq. (A18), we now find for Eq. (A19)

$$
\mathbf{M}_{y l}^{-1} \mathbf{J}_{l} \check{\mathbf{M}}_{x l}^{-1}=\mathbf{T}_{y} \mathbf{R}_{y l}^{-1} \mathbf{C}_{\boldsymbol{I}} \check{\mathbf{R}}_{x l}^{-1} \mathbf{T}_{x}^{-1} \sqrt{\beta_{x l} \beta_{y l}} \tilde{\dot{j}}_{l},
$$

and therefore we can write

$$
\begin{aligned}
\mathbf{m}+\overline{\mathbf{n}}= & \mathbf{T}_{y} \sum_{l=1}^{N_{l}}\left(\check{\mathbf{R}}_{y l} \mathbf{C}_{\boldsymbol{I}} \mathbf{R}_{x l}-\mathbf{R}_{y l}^{-1} \mathbf{C}_{\boldsymbol{I}} \check{\mathbf{R}}_{x l}^{-1}\right) \sqrt{\beta_{x l} \beta_{y l}} \tilde{j}_{l} \mathbf{T}_{x}^{-1} \\
& +O(2) .
\end{aligned}
$$

Now note that $\check{\mathbf{R}}_{y l}=\mathbf{R}_{y} \mathbf{R}_{y l}^{-1}$, and $\check{\mathbf{R}}_{x l}^{-1}=\mathbf{R}_{x l} \mathbf{R}_{x}^{-1}$, where $\mathbf{R}_{x}, \mathbf{R}_{y}$ are the matrices of the one-turn map (in the Courant-Snyder reference frame). Therefore we can write

$$
\begin{aligned}
\mathbf{m}+\overline{\mathbf{n}}= & \mathbf{T}_{\boldsymbol{y}} \sum_{l=1}^{N_{l}} \mathbf{R}_{y l}^{-1}\left(\mathbf{R}_{y} \mathbf{C}_{\boldsymbol{I}}-\mathbf{C}_{\boldsymbol{I}} \mathbf{R}_{x}^{-1}\right) \mathbf{R}_{x l} \sqrt{\beta_{x l} \beta_{y l}} \tilde{j}_{l} \mathbf{T}_{x}^{-1} \\
& +O(2)
\end{aligned}
$$

so that

$$
\begin{aligned}
|\mathbf{m}+\overline{\mathbf{n}}|= & \left|\sum_{l=1}^{N_{l}} \mathbf{R}_{y l}^{-1}\left(\mathbf{R}_{y} \mathbf{C}_{\boldsymbol{I}}-\mathbf{C}_{\boldsymbol{I}} \mathbf{R}_{x}^{-1}\right) \mathbf{R}_{x l} \sqrt{\beta_{x l} \beta_{y l}} \tilde{j}_{l}\right| \\
& +O(3)
\end{aligned}
$$

as $\left|\mathbf{T}_{\boldsymbol{x}}\right|=\left|\mathbf{T}_{\boldsymbol{y}}\right|=1$. Finally, defining the matrix

$$
\mathcal{M}^{l}=\mathbf{R}_{y l}^{-1}\left(\mathbf{R}_{y} \mathbf{C}_{I}-\mathbf{C}_{I} \mathbf{R}_{x}^{-1}\right) \mathbf{R}_{x l}
$$

we write the final form

$$
\begin{aligned}
|\mathbf{m}+\overline{\mathbf{n}}|= & \sum_{l t} \sqrt{\beta_{x l} \beta_{y l} \beta_{x t} \beta_{y t}}\left(\mathcal{M}_{11}^{l} \mathcal{M}_{22}^{t}-\mathcal{M}_{12}^{l} \mathcal{M}_{21}^{t}\right) \tilde{j}_{l} \tilde{j}_{t} \\
& +O(3)
\end{aligned}
$$

Note that for a lattice with only one error, for example, located at $s=s_{l}$ we find $\left|\mathcal{M}^{l}\right|=\sin \left(2 \pi Q_{x 0}\right) \sin \left(2 \pi Q_{y 0}\right)$.

\section{Perturbative expansion}

In the previous sections we have derived that

$$
\begin{aligned}
\Lambda_{+}= & 2 \cos \left(2 \pi Q_{x 0}\right)-\sin \left(2 \pi Q_{x 0}\right) \sum_{l=1}^{N_{l}} \beta_{x l} \tilde{k}_{l} \\
& +\sum_{l, q=1}^{N_{l}} \mathcal{K}_{l q}^{x} \tilde{k}_{l} \tilde{k}_{q}+\sum_{l, q=1}^{N_{l}} \mathcal{J}_{l q}^{x} \tilde{j}_{l} \tilde{j}_{q}+O(3), \\
\Lambda_{-}= & 2 \cos \left(2 \pi Q_{y 0}\right)+\sin \left(2 \pi Q_{y 0}\right) \sum_{l=1}^{N_{l}} \beta_{y l} \tilde{k}_{l} \\
& +\sum_{l, q=1}^{N_{l}} \mathcal{K}_{l q}^{y} \tilde{k}_{l} \tilde{k}_{q}+\sum_{l, q=1}^{N_{l}} \mathcal{J}_{l q}^{y} \tilde{j}_{l} \tilde{j}_{q}+O(3),
\end{aligned}
$$

where

$$
\begin{aligned}
\mathcal{K}_{l q}^{x}= & \frac{1}{2} \beta_{x l} \beta_{x q} \mathcal{S}_{x,[l q]} \check{\mathcal{S}}_{x,[l q]}, \\
\mathcal{J}_{l q}^{x}= & \frac{1}{2} \sqrt{\beta_{x l} \beta_{y l} \beta_{x q} \beta_{y q}} \mathcal{S}_{y,[l q]} \check{\mathcal{S}}_{x,[l q]} \\
& +\frac{\sqrt{\beta_{x l} \beta_{y l} \beta_{x q} \beta_{y q}}}{2\left[\cos \left(2 \pi Q_{x 0}\right)-\cos \left(2 \pi Q_{y 0}\right)\right]} \\
& \times\left(\mathcal{M}_{11}^{l} \mathcal{M}_{22}^{q}-\mathcal{M}_{12}^{l} \mathcal{M}_{21}^{q}\right), \\
\mathcal{K}_{l q}^{y}= & \frac{1}{2} \beta_{y l} \beta_{y q} \mathcal{S}_{y,[l q]} \check{\mathcal{S}}_{y,[l q]}, \\
\mathcal{J}_{l q}^{y}= & \frac{1}{2} \sqrt{\beta_{x l} \beta_{y l} \beta_{x q} \beta_{y q}} \mathcal{S}_{x,[l q]} \check{\mathcal{S}}_{y,[l q]} \\
& -\frac{\sqrt{\beta_{x l} \beta_{y l} \beta_{x q} \beta_{y q}}}{2\left[\cos \left(2 \pi Q_{x 0}\right)-\cos \left(2 \pi Q_{y 0}\right)\right]} \\
& \times\left(\mathcal{M}_{11}^{l} \mathcal{M}_{22}^{q}-\mathcal{M}_{12}^{l} \mathcal{M}_{21}^{q}\right) .
\end{aligned}
$$

From the properties of $\mathcal{S}_{x,[i j]}, \check{\mathcal{S}}_{x,[i j]}, \mathcal{S}_{y,[i j]}, \check{\mathcal{S}}_{y,[i j]}$, we obtain that $\mathcal{K}_{l q}^{x}=\mathcal{K}_{q l}^{x}$, and $\mathcal{K}_{l q}^{y}=\mathcal{K}_{q l}^{y}$. We also find that $\mathcal{K}_{l l}^{x}=\mathcal{K}_{l l}^{y}=0$ for $l=1, \ldots, N_{l}$. We now recall that $\quad \Lambda_{+}=2 \cos \left[2 \pi\left(Q_{x 0}+\Delta Q_{x}\right)\right]$, and $\Lambda_{-}=$ $2 \cos \left[2 \pi\left(Q_{y 0}+\Delta Q_{y}\right)\right]$, and considering the expansion

$$
\begin{aligned}
& \Delta Q_{x}=\sum_{l=1}^{N_{l}} \tilde{\mathcal{K}}_{l}^{x} \tilde{k}_{l}+\sum_{l, q=1}^{N_{l}} \tilde{\mathcal{K}}_{l q}^{x} \tilde{k}_{l} \tilde{k}_{q}+\sum_{l, q=1}^{N_{l}} \tilde{\mathcal{J}}_{l q}^{x} \tilde{j}_{l} \tilde{j}_{q}+O(3), \\
& \Delta Q_{y}=\sum_{l=1}^{N_{l}} \tilde{\mathcal{K}}_{l}^{y} \tilde{k}_{l}+\sum_{l, q=1}^{N_{l}} \tilde{\mathcal{K}}_{l q}^{y} \tilde{k}_{l} \tilde{k}_{q}+\sum_{l, q=1}^{N_{l}} \tilde{\mathcal{J}}_{l q}^{y} \tilde{j}_{l} \tilde{j}_{q}+O(3),
\end{aligned}
$$

by expanding the expression of $\Lambda_{ \pm}$and equating the terms of each order we find

$$
\begin{aligned}
\tilde{\mathcal{K}}_{l}^{x} & =+\frac{\beta_{x l}}{4 \pi}, \\
\tilde{\mathcal{K}}_{l q}^{x} & =-\frac{1}{4 \pi \sin \left(2 \pi Q_{x 0}\right)}\left[\mathcal{K}_{l q}^{x}+\frac{\beta_{x l} \beta_{x q}}{4} \cos \left(2 \pi Q_{x 0}\right)\right] \\
\tilde{\mathcal{J}}_{l q}^{x} & =-\frac{\mathcal{J}_{l q}^{x}}{4 \pi \sin \left(2 \pi Q_{x 0}\right)},
\end{aligned}
$$


and

$\tilde{\mathcal{K}}_{l}^{y}=-\frac{\beta_{y l}}{4 \pi}$

$\tilde{\mathcal{K}}_{l q}^{y}=-\frac{1}{4 \pi \sin \left(2 \pi Q_{y 0}\right)}\left[\mathcal{K}_{l q}^{y}+\frac{\beta_{y l} \beta_{y q}}{4} \cos \left(2 \pi Q_{y 0}\right)\right]$,

$\tilde{\mathcal{J}}_{l q}^{y}=-\frac{\mathcal{J}_{l q}^{y}}{4 \pi \sin \left(2 \pi Q_{y 0}\right)}$.

As $\mathcal{K}_{l q}^{x}, \mathcal{K}_{l q}^{y}$ are symmetric, then $\tilde{\mathcal{K}}_{l q}^{x}, \tilde{\mathcal{K}}_{l q}^{y}$ are symmetric as well.

\section{Nonlinear tune response matrix}

Now we characterize $\tilde{k}_{l}, \tilde{j}_{l}$ by inserting Eqs. (14) into the expressions (A22) and find the contribution of the nonlinear components to the tune measured with respect the closed orbit. Explicitly the terms to be computed are

$$
\begin{aligned}
\sum_{l=1}^{N_{l}} \tilde{\mathcal{K}}_{l}^{x} \tilde{k}_{l}= & \sum_{l=1}^{N_{l}} \tilde{\mathcal{K}}_{l}^{x}\left[K_{1 l}+K_{2 l} x_{o l}-J_{2 l} y_{o l}\right. \\
& \left.+K_{3 l} \frac{1}{2}\left(x_{o l}^{2}-y_{o l}^{2}\right)-J_{3 l} x_{o l} y_{o l}\right]
\end{aligned}
$$

and

$$
\begin{aligned}
\sum_{l, q=1}^{N_{l}} \tilde{\mathcal{K}}_{l q}^{x} \tilde{k}_{l} \tilde{k}_{q}= & \sum_{l, q=1}^{N_{l}} \tilde{\mathcal{K}}_{l q}^{x}\left[K_{1 l}+K_{2 l} x_{o l}-J_{2 l} y_{o l}\right. \\
& \left.+K_{3 l} \frac{1}{2}\left(x_{o l}^{2}-y_{o l}^{2}\right)-J_{3 l} x_{o l} y_{o l}\right] \\
& \times\left[K_{1 q}+K_{2 q} x_{o q}-J_{2 q} y_{o q}\right. \\
& \left.+K_{3 q} \frac{1}{2}\left(x_{o q}^{2}-y_{o q}^{2}\right)-J_{3 q} x_{o q} y_{o q}\right]
\end{aligned}
$$

and

$$
\begin{aligned}
\sum_{l, q=1}^{N_{l}} \tilde{\mathcal{J}}_{l q}^{x} \tilde{j}_{l} \tilde{j}_{q}= & \sum_{l, q=1}^{N_{l}} \tilde{\mathcal{J}}_{l q}^{x}\left[K_{2 l} y_{o l}+J_{2 l} x_{o l}+K_{3 l} x_{o l} y_{o l}\right. \\
& \left.+J_{3 l} \frac{1}{2}\left(x_{o l}^{2}-y_{o l}^{2}\right)\right] \\
& \times\left[K_{2 q} y_{o q}+J_{2 q} x_{o q}+K_{3 q} x_{o q} y_{o q}\right. \\
& \left.+J_{3 q} \frac{1}{2}\left(x_{o q}^{2}-y_{o q}^{2}\right)\right] .
\end{aligned}
$$

As the closed orbit at the location of the errors [23] is

$$
x_{o l}=\sum_{t=1}^{N_{t}} M_{l t}^{x} \theta_{x t}, \quad y_{o l}=\sum_{t=1}^{N_{t}} M_{l t}^{y} \theta_{y t},
$$

we can substitute Eqs. (A28) into Eqs. (A25)-(A27), and find the dependence of $\Delta Q_{x}, \Delta Q_{y}$ from the steerer angle. We find then

$$
\begin{aligned}
\Delta Q_{x}= & { }_{x} Q+\sum_{t=1}^{N_{t}}\left({ }_{x} Q_{t}^{x} \theta_{x t}+{ }_{x} Q_{t}^{y} \theta_{y t}\right) \\
& +\sum_{t, i=1}^{N_{t}}\left({ }_{x} Q_{t i}^{x x} \theta_{x t} \theta_{x i}+{ }_{x} Q_{t i}^{y y} \theta_{y t} \theta_{y i}+{ }_{x} Q_{t i}^{x y} \theta_{x t} \theta_{y i}\right)
\end{aligned}
$$

$$
\begin{aligned}
\Delta Q_{y}= & { }_{y} Q+\sum_{t=1}^{N_{t}}\left({ }_{y} Q_{t}^{x} \theta_{x t}+{ }_{y} Q_{t}^{y} \theta_{y t}\right) \\
& +\sum_{t, i=1}^{N_{t}}\left({ }_{y} Q_{t i}^{x x} \theta_{x t} \theta_{x i}+{ }_{y} Q_{t i}^{y y} \theta_{y t} \theta_{y i}+{ }_{y} Q_{t i}^{x y} \theta_{x t} \theta_{y i}\right),
\end{aligned}
$$

where

$$
\begin{aligned}
& { }_{x} Q=\sum_{l=1}^{N_{l}} K_{1 l} \tilde{\mathcal{K}}_{l}^{x}+\sum_{l, q=1}^{N_{l}} K_{1 l} K_{1 q} \tilde{\mathcal{K}}_{l q}^{x}, \\
& { }_{x} Q_{t}^{x}=\sum_{l=1}^{N_{l}} K_{2 l} \tilde{\mathcal{K}}_{l}^{x} M_{l t}^{x}+\sum_{l, q=1}^{N_{l}} K_{1 q} K_{2 l}\left(\tilde{\mathcal{K}}_{l q}^{x}+\tilde{\mathcal{K}}_{q l}^{x}\right) M_{l t}^{x}, \\
& { }_{x} Q_{t}^{y}=-\sum_{l=1}^{N_{l}} J_{2 l} \tilde{\mathcal{K}}_{l}^{x} M_{l t}^{y}+\sum_{l, q=1}^{N_{l}}\left[-K_{1 q} J_{2 l}\left(\tilde{\mathcal{K}}_{l q}^{x}+\tilde{\mathcal{K}}_{q l}^{x}\right)\right] M_{l t}^{y}, \\
& { }_{x} Q_{t i}^{x x}=\frac{1}{2} \sum_{l=1}^{N_{l}} K_{3 l} \tilde{\mathcal{K}}_{l}^{x} M_{l t}^{x} M_{l i}^{x} \\
& +\frac{1}{2} \sum_{l, q=1}^{N_{l}}\left[K_{1 q} K_{3 l}\left(\tilde{\mathcal{K}}_{l q}^{x}+\tilde{\mathcal{K}}_{q l}^{x}\right)\right] M_{l t}^{x} M_{l i}^{x} \\
& +\sum_{l, q=1}^{N_{l}}\left(J_{2 l} J_{2 q} \tilde{\mathcal{J}}_{l q}^{x}+K_{2 l} K_{2 q} \tilde{\mathcal{K}}_{l q}^{x}\right) M_{l t}^{x} M_{q i}^{x} \text {, } \\
& { }_{x} Q_{t i}^{y y}=-\frac{1}{2} \sum_{l=1}^{N_{l}} K_{3 l} \tilde{\mathcal{K}}_{l}^{x} M_{l t}^{y} M_{l i}^{y} \\
& -\frac{1}{2} \sum_{l, q=1}^{N_{l}}\left[K_{1 q} K_{3 l}\left(\tilde{\mathcal{K}}_{l q}^{x}+\tilde{\mathcal{K}}_{q l}^{x}\right)\right] M_{l t}^{y} M_{l i}^{y} \\
& +\sum_{l, q=1}^{N_{l}}\left(K_{2 l} K_{2 q} \tilde{\mathcal{J}}_{l q}^{x}+J_{2 l} J_{2 q} \tilde{\mathcal{K}}_{l q}^{x}\right) M_{l t}^{y} M_{q i}^{y}, \\
& { }_{x} Q_{t i}^{x y}=-\sum_{l=1}^{N_{l}} J_{3 l} \tilde{\mathcal{K}}_{l}^{x} M_{l t}^{x} M_{l i}^{y} \\
& +\sum_{l, q=1}^{N_{l}}\left[-K_{1 q} J_{3 l}\left(\tilde{\mathcal{K}}_{l q}^{x}+\tilde{\mathcal{K}}_{q l}^{x}\right)\right] M_{l t}^{x} M_{l i}^{y} \\
& +\sum_{l, q=1}^{N_{l}}\left[J_{2 l} K_{2 q}\left(\tilde{\mathcal{J}}_{l q}^{x}+\tilde{\mathcal{J}}_{q l}^{x}\right)\right. \\
& \left.-J_{2 q} K_{2 l}\left(\tilde{\mathcal{K}}_{l q}^{x}+\tilde{\mathcal{K}}_{q l}^{x}\right)\right] M_{l t}^{x} M_{q i}^{y} .
\end{aligned}
$$


In case $K_{1 l}$ are small compared with the sextupolar and octupolar components, we obtain Eqs. (20). The nonlinear tune matrix elements for $\Delta Q_{y}$ namely ${ }_{y} Q_{t}^{x},{ }_{y} Q_{t}^{y},{ }_{y} Q_{t t}^{x x}$, ${ }_{y} Q_{t t}^{y y},{ }_{y} Q_{t t}^{x y}$ are readily obtained from Eqs. (A31) with the following substitutions: $\tilde{\mathcal{K}}_{l}^{x} \rightarrow \tilde{\mathcal{K}}_{l}^{y}, \quad \tilde{\mathcal{K}}_{l q}^{x} \rightarrow \tilde{\mathcal{K}}_{l q}^{y}$, and $\tilde{\mathcal{J}}_{l q}^{x} \rightarrow \tilde{\mathcal{J}}_{l q}^{y}$.

Note that the derivation of Eqs. (A31) is based on Eq. (A1), that is the natural linear coupling terms $J_{1 l}$ are absent, so that the orbit response at the location of the errors is given by Eqs. (A28). The effect of a small linear coupling would create in Eqs. (A27), in addition to a correction in Eq. (A1), small correction terms proportional to $J_{1 l} J_{1 q}, J_{1 l} K_{2 q}, J_{1 l} J_{2 q}, J_{1 l} K_{3 q}$, and $J_{1 l} J_{3 q}$, which therefore becomes negligible if $\left|J_{1 l}\right|$ is smaller than $\left|K_{2 l}\right|,\left|J_{2 l}\right|$, $\left|K_{3 l}\right|,\left|J_{3 l}\right|$. A discussion on a method for correcting the natural linear coupling and its applications can be found in Ref. [18].

[1] G. Franchetti et al., Phys. Rev. ST Accel. Beams 6, 124201 (2003); G. Franchetti and I. Hofmann, in Proceedings of the 39th ICFA Advanced Beam Dynamics Workshop: High Intensity High Brightness Hadron Beams, Tsukuba, 2006, p. 167.

[2] A. Schoch, Report No. CERN 57-21, 1958; G. Guignard, Report No. CERN 78-11, 1978.

[3] A. Bazzani, E. Todesco, G. Turchetti, and G. Servizi, Report No. CERN 94-02, 1994.

[4] W. Fischer, M. Giovannozzi, and F. Schmidt, Phys. Rev. E 55, 3507 (1997).

[5] O. Brüning, W. Fischer, F. Schmidt, and F. Willeke, Report No. CERN-SL 95-69 AP, 1995.
[6] F. Zimmermann, Report No. SLAC-PUB-6504, 1994.

[7] J. Bengtsson, Report No. CERN 88-05, 1988.

[8] R. Bartolini and F. Schmidt, Part. Accel. 59, 93 (1998).

[9] R. Tomás et al., Phys. Rev. ST Accel. Beams 8, 024001 (2005).

[10] J. Laskar, C. Froeschlé, and A. Celletti, Physica (Amsterdam) 56D, 253 (1992); R. Bartolini et al., Part. Accel. 56, 167 (1996).

[11] G. Arduini et al., in Proceedings of the Particle Accelerator Conference, Portland, OR, 2003 (IEEE, New York, 2003), p. 2240.

[12] R. Cappi et al., in Ref. [11], p. 2913.

[13] M. Berz et al., Nucl. Instrum. Methods Phys. Res., Sect. A 427, 310 (1999); M. Berz, Modern Map Methods in Particle Beam Physics (Academic Press, New York, 1999).

[14] J-P. Koutchouk, F. Pilat, and V. Ptistyn, in Proceedings of the Particle Accelerator Conference, Chicago, IL, 2001 (IEEE, New York, 2001), p. 1684.

[15] F. Pilat et al., in Proceedings of the 2005 Particle Accelerator Conference, Knoxville, Tennessee, USA, 2005, p. 601.

[16] J. Safranek, Nucl. Instrum. Methods Phys. Res., Sect. A 388, 27 (1997).

[17] E. D. Courant and H. S. Snyder, Ann. Phys. (Paris) 3, 1 (1958).

[18] Wolfram Fischer, Phys. Rev. ST Accel. Beams 6, 062801 (2003).

[19] J. Bengtsson and J. Irwin, SSC Laboratory, February 1990.

[20] G. Franchetti, I. Hofmann, and G. Turchetti, AIP Conf. Proc. 448, 233 (1998).

[21] R. Bartolini et al., Part. Accel. 52, 147 (1996).

[22] C. J. Gardner, BNL Report No. C-A/AP/\#101, 2003.

[23] These equations are equal to Eqs. (5), but for convenience of the reader we rewrite them here. 